\title{
Die Bedeutung des Ausdrucks ,am Tag ...“ in Genesis 2,4 im Rahmen des Pentateuch
}

\author{
SIEGBERT RIECKER (ETF LEUVEN, BELGIUM) \\ AND HENDRIK J. KoOREVAAR (ETF LEUVEN, BELGIUM)
}

\begin{abstract}
The interpretation of bojôm in Gen 2:4 as "when" in the sense of an unspecific period of time has often been used as an argument to defend an allegorical or figurative interpretation of the days of creation. A comparison with parallel grammatical constructions throughout the Pentateuch casts severe doubts on that idea, which are confirmed by a closer exegetical analysis of bəjôm in Gen 2:4b in its individual literary contexts (Gen 2:4a, 1:1, 2:5-3:24, especially the parallel narrative sequence following 5:1-2). Without "Systemzwang," all arguments point to the natural understanding of bojôm in Gen 2:4 as "in the day," referring to a specific day, the first day of creation.
\end{abstract}

KEYWORDS: Biblical days, וֹ, creation, infinitivus constructus, Gen. 1:1, Gen. 2:4b

\section{A EINFÜHRUNG UND METHODE}

Nicht selten bleibt in der Debatte über die Bedeutung des Ausdrucks i jôm „Tag“ in Gen 1,1-2,3 unberücksichtigt, dass der Ausdruck ein weiteres Mal in Gen 2,4 auftaucht: „Dies sind die Toledot des Himmels und der Erde, als sie geschaffen wurden, מבְּיוֹ bajôm macht JHWH Gott Erde und Himmel.“

Dass die Mehrzahl gängiger deutscher Übersetzungen בִּיוֹ bojôm (wörtlich „am Tag“) durch „zu der (zur) Zeit“" wiedergibt, während King James und englische Übersetzungen überwiegend dem wörtlichen Verständnis in

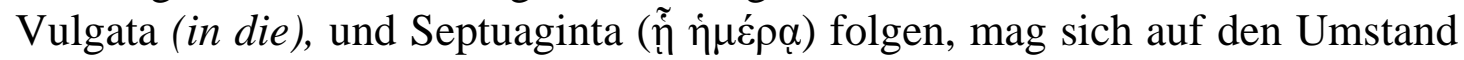
zurückführen lassen, dass bereits Luther in seiner Genesis-Vorlesung im Jahr 1535 erklärt: „Daß er spricht: ,Zu der Zeit', ist zu verstehen von einer Zeit, so da noch währete und ungeendet war. Als wollte er sagen: Zur selben Zeit stund es mit allen Dingen sehr wohl und war das schönste Wesen in der Welt". ${ }^{\text {‘ }}$

\footnotetext{
* Submitted: 24/07/2019; peer-reviewed: 22/01/2020; accepted: 22/01/2020. Siegbert Riecker and Hendrik J. Koorevaar, "Die Bedeutung des Ausdrucks "am Tag ... in Genesis 2,4 im Rahmen des Pentateuch", Old Testament Essays 33 no. 1 (2020): 80 - 106. DOI: https://doi.org/10.17159/2312-3621/2020/v33n1a6.

1 WA 42, 62, 30-31, Übersetzung Johann Georg Walch in Martin Luther, dr Martin Luthers Auslegung des ersten Buches Mose: Erster Teil (St. Louis: Concordia, 1880), 101.
} 
Eine Festigung ${ }^{2}$ dieses Verständnisses in der Kommentarliteratur bis heute $^{3}$ wurde begünstigt durch eine Bemerkung von Magne Sæbø im in seinem einflussreichen Artikel im Theologischen Wörterbuch zum Alten Testament:

Die Bedeutung, Tag' wird mehr oder weniger geschwächt, wenn ein Präpositionalausdruck mit jôm (gelegentlich auch $j^{e} m \hat{e}$ ) noch mit einem Verb verbunden ist. An erster Stelle steht hier $b^{e}$ jôm mit Inf. (fast 70mal) im Sinne einer allgemeinen Zeitangabe bzw. einer temporalen Konjunktion ,als/wenn', jedoch ohne daß die Grundbedeutung ,Tag' völlig ausgeschlossen zu sein braucht (zur wichtigen Stelle Gen 2,4b nach dem vorangehenden 7-TageSchema vgl. C. Westermann, Genesis, BK I/1, $\left.{ }^{2} 1976,270\right) .{ }^{4}$

Sæbø hebt Genesis 2,4b hier als Beweis oder besonderes Beispiel dafür hervor, dass בִּיוֹס bajôm mit Infinitiv in seiner wörtlichen Bedeutung geschwächt und zu einer Konjunktion ,,als/wenn“" werden kann.

Ist die Wendung in Gen 2,4 als allgemeiner Hinweis auf die „Zeit““ der Weltschöpfung zu verstehen, wird das Wort „Tag“ für einen längeren Zeitraum als 24 Stunden verwendet. Dies wiederum wird regelmäßig als Argument dafür genutzt, dass das Wort „Tag“ auch in Gen 1,1-2,3 etwas anderes als einen 24Stunden-Tag bezeichne. ${ }^{5}$

2 Vgl. ältere Kommentare August Knobel, Die Genesis erklärt (Leipzig: Weidmann 1852), 23: „zur Zeit“; August Dillmann, Die Genesis (Leipzig: Hirzel, 1892), 51: „zur Zeit da“; Karl Marti, Genesis (Tübingen: J.C.B. Mohr, 1898), 24: „als“; Hermann Gunkel, Genesis (3. Aufl.; Göttingen: Vandenhoeck \& Ruprecht, 1922), 4: „zur Zeit da“; John Skinner, Genesis (New York: Scribner, 1910), 54: „At the time when"; darüber hinaus Wilhelm Gesenius, Handwörterbuch über das Alte Testament (Hg. F. Buhl; 17. Aufl.; Berlin: Springer, 1962), 293: „zu der Zeit wo = wie “; Gerhard von Rad, Das erste Buch Mose: Genesis (8. Aufl.; Göttingen: Vandenhoeck \& Ruprecht, 1967), 58: „als“; anders jedoch Karl Budde, Die Biblische Urgeschichte (Gen. 1-12,5) (Gießen: Ricker, 1883), 522: „Am Tage, da“ und wohl auch Carl Friedrich Keil, Genesis und Exodus (Leipzig: Dörffling und Francke, 1878), 47.

3 Vgl. die jüngsten deutschen Kommentare sämtlich mit ,als“: Andreas Schüle, Die Urgeschichte (Genesis 1-11) (Zürich: TVZ, 2009), 52; Jan Christian Gertz, Das erste Buch Mose. Genesis: Die Urgeschichte. Gen 1-11 (Göttingen: Vandenhoeck \& Ruprecht, 2018), 80; Georg Fischer, Genesis 1-11 (Freiburg: Herder, 2018), 174.

4 Magne Sæbø in Jan Bergman, u.a., „יוֹ yôm“, ThWAT 3: 568. Vgl. Pieter

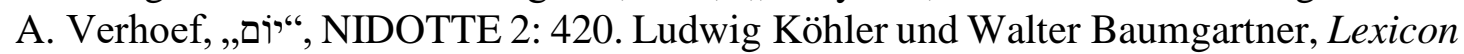
in Veteris Testamenti Libros (Leiden: Brill, 1985), 373. Bruce K. Waltke und Michael P. O'Conner, An Introduction to Biblical Hebrew Syntax (Winona Lake: Eisenbrauns, 1990), 250, 611.

$5 \quad$ Vgl. zusammenfassend zum weitgehenden kritischen Forschungskonsens einer Deutung der Tage in Genesis 1 als „normale“ 24-Stunden-Tage u.a. Terence E. Fretheim, „Were the Days of Creation Twenty-Four Hours Long? Yes“, in The Genesis Debate: Persistent Questions About Creation and the Flood (Hg. R. Youngblood; 2. 
Kann das Wort „Tag“ im Singular eine Zeit unbestimmter Dauer bedeuten, und wenn dem so ist, was bedeutet es dann in Gen 2,4? Angenommen, „Tag“" in Gen 2,4 bezeichne wirklich eine längere Periode, würde dann ein Zusammenhang zu dem Wort „Tag“ in Gen 1,1-2,3, welches sich nur auf einzelne Abschnitte des Schöpfungswerkes bezieht, nicht an Wahrscheinlichkeit verlieren ${ }^{6}$

Es geht hier nicht darum, alle Bedeutungen und Funktionen des Wortes „Tag“ im Alten Testament zu untersuchen, ${ }^{7}$ sondern lediglich, das hebräische Wort für „Tag" grammatisch und semantisch in seinem speziellen Kontext in Gen 2,4 zu verstehen. Begünstigt wird dieses Unternehmen dadurch, dass im Hebräischen eine besondere Konstruktion verwendet wird: עִ עִשוֹת bajôm 'ăśôt, wörtlich: ,,in - Tag - machen“. Dem Wort םi' jôm „Tag“ geht die Vorsilbe $\underset{\text { ב }}{\text { ב }}$ o ,in“ voraus, worauf ein Verb im Infinitiv constructus folgt. ${ }^{8}$ Diese Konstruktion, diese Kombination, soll hier untersucht werden, beschränkt auf den Pentateuch. Eine umfangreichere Untersuchung aller beinahe 70 Belege im gesamten Alten Testament ${ }^{9}$ würde den hier gegebenen Rahmen sprengen.

Aufl.; Eugene: Wipf and Stock, 1999), 12-35. Zur Geschichte allegorischer Deutungen der Schöpfungstage und Deutungen auf/als lange Zeiträume vgl. Jack P. Lewis, „The Days of Creation. An Historical Survey of Interpretation“, JETS 32 (1989): 433-455, Gerhard F. Hasel, „The ,Days' of Creation in Genesis 1: Literal ,Days“ or Figurative ,Periods/Epochs“ of Time?“, Origins 21 (1994): 5-38. Walter Hilbrands, „Die Länge der Schöpfungstage: Eine exegetische und rezeptionsgeschichtliche Untersuchung von יוֹ (,Tag“) in Gen 1,1-2,3“, BN N.F. 149 (2011): 3-5. Walter M. Booth, „,Days of Genesis 1: Literal or Nonliteral?“, JATS 14 (2003): 101-120; James B. Jordan, Creation in Six Days: A Defense of the Traditional Reading of Genesis One (Moscow: Canon, 1999).

6 Vgl. ähnlich David M. Fouts, „Selected Lexical and Grammatical Studies in Genesis 1“, AUSS 42 (2004): 81, 85: Ein idiomatisch gebrauchter Begriff kann kein Argument liefern für das Verständnis des Begriffs außerhalb einer Redewendung.

7 So etwa Gerhard von Rad, „,Der Tag“ im AT“, ThWNT 2: 945-949. Ernst Jenni, „יוֹ jōm Tag“, THAT 1: 707-726. Bergman, „yôm“, 3: 566-586. Verhoef, „יוֹ,“, 2: 419424. Bob Becking, „Day יום,“, in Dictionary of Deities and Demons in the Bible (Hg. Karel van der Toorn, u.a.; 2. Aufl.; Leiden: Brill, 1999), 221-223. Jörg Lanckau, „Tag / Tageszeiten (AT)“, Das Wissenschaftliche Lexikon im Internet (WiBiLex) [zitiert 14.5.2019], (Hg. Stefan Alkier u.a.). Online: http://www.bibelwissenschaft.de /stichwort/32270. Edward J. Young, „The Days of Genesis“, WTJ 25 (1963): 1-34, 143 171. Gershon Brin, ,The Formula X- יוים Some Characteristics of Historiographical Writing in Israel“, ZAW 93 (1981): 183-196. Ders., The Concept of Time in the Bible and the Dead Sea Scrolls (Leiden: Brill, 2001), 52-58, 78-93, 153166.

8 Parallelen im Plural (Rut 1,1; 2 Chr 26,5) deuten darauf hin, dass das Wort יוֹ jôm in bọ bojôm in Gen 2,4 im status constructus steht (im Sg. formal nicht erkennbar).

9 Vgl. Jenni, ,jōm“, 711; John R. Wilch, Time and Event: An Exegetical Study of the Use of 'eth in the Old Testament in Comparison to Other Temporal Expressions in 
Genesis ist als Teil des „Triptychon“" der Tora eng mit dem folgenden Buch Exodus-Levitikus-Numeri (dem „Mittelstück“ der Tora) verbunden. ${ }^{10}$

Da sich das überkommene JEDP-Paradigma seit den späten 1970er Jahren in einer Krise befindet und hinsichtlich des Umfangs und der Datierung postulierter Quellen und Bearbeitungen bzw. Kompositionsschichten keine neuer Konsens in Sichtweite ist, wäre es methodisch inkonsequent, bestimmte der uns gemeinsam als Tora überlieferten Texte apriorisch von der Untersuchung auszuschließen. Sowohl bei der Pentateuch- bzw. Hexateuch-Hypothese (Genesis-Josua) ${ }^{11}$ als auch bei einem historisch-kanonischen Ansatz liegt die Datierung der Endredaktionen der Bücher Genesis bis Deuteronomium zudem nahe beieinander, wenn auch mit einem Unterschied von beinahe 1000 Jahren, in persischer oder mosaisch-josuanischer Zeit. ${ }^{12}$ Die Endredaktionen stammen grammatisch und semantisch betrachtet auch aus derselben Zeit, was nicht ausschließt, dass ihre Quellen viel älter sein können, vor allem die der Genesis. Die Quellen mögen sich inhaltlich unterscheiden, doch in der Endredaktion sind sie zu einer Einheit verschmolzen. Unter Einschluss des Buchs Deuteronomium

Clarification of the Concept of Time (Leiden: Brill, 1969), 91; Bryan C. Hodge, Revisiting the Days of Genesis. A Study of the Use of Time in Genesis 1-11 in Light of its Ancient Near Eastern and Literary Context (Eugene: Wipf and Stock, 2011), 80.

$10 \mathrm{Vgl}$. zur These eines drei-statt fünfgliedrigen Aufbaus der Tora und der literarischen Einheit der Bücher Exodus bis Numeri Hendrik J. Koorevaar, „The Books of Exodus - Leviticus - Numbers and the Macro-Structural Problem of the Pentateuch", in The Books of Leviticus and Numbers (Hg. T. Römer; Leuven: Peeters, 2008), 423453. Ders., „Steps for Dating the Books of the Pentateuch: a literary and historical canonical approach", in Paradigm Change in Pentateuchal Research. Tagung vom 16.18. März 2017 an der STH Basel (Hg. Matthias Armgardt u.a.; Wiesbaden: Harrassowitz, 2019), 227-242.

11 Vgl. einführend zur Frage einer Pentateuch- oder (möglicherweise gleichzeitig stattfindenden) Hexateuch-Edition in persischer Zeit Christophe Nihan und Thomas Römer, „Die Entstehung des Pentateuch: Die aktuelle Debatte“, in: Einleitung in das Alte Testament: Die Bücher der Hebräischen Bibel und die alttestamentlichen Schriften der katholischen, protestantischen und orthodoxen Kirche (Hg. Thomas Römer u.a.) (Zürich: TVZ, 2013), 158-160. Der dort genannte Reinhard Achenbach, Die Vollendung der Tora: Studien zur Rechtsgeschichte des Numeribuches im Kontext von Hexateuch und Pentateuch (Wiesbaden: Harrassowitz, 2003), 629 bestätigt die These von Eckart Otto, Das Deuteronomium im Pentateuch und Hexateuch: Studien zur Literaturgeschichte von Pentateuch und Hexateuch im Lichte des Deuteronomiumrahmens (Tübingen: Mohr, 2000), wonach sich an die zwei Phasen einer Hexateuch- (1. Hälfte des 5. Jh.) und Pentateuchredaktion (2. Hälfte des 5. Jh.) die dritte Phase einer Theokratischen Bearbeitung anschließt (4. Jh. v.Chr.).

12 Vgl. einführend Cornelis Houtman, Der Pentateuch: Die Geschichte seiner Erforschung neben einer Auswertung (Kampen: Kok, 1994), 354-365 zum Selbstzeugnis des Pentateuch, sowie dem Maß an Offenheit verschiedener Vertreter eines historisch-kanonischen Ansatzes gegenüber post-mosaischer Bearbeitung. 
wird unsere Untersuchung genügend Material zutage fördern, auf dessen Basis fundierte Schlussfolgerungen möglich sind.

Es bleibt hier auch kein Raum, Kombinationen mit einer anderen Vorsilbe als $\underline{b} \not \underline{b}$, ,in“ zu untersuchen, oder Kombinationen des bestimmten Artikels mit

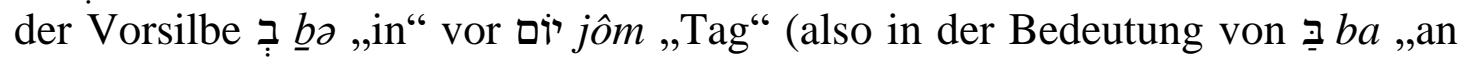
dem") oder Kombinationen von בִּיוֹס bajôm ,am Tag“" mit Substantiv ${ }^{13}$ oder Adjektiv. ${ }^{14}$ Solche Untersuchungen wären sehr umfangreich und würden sicherlich lohnende Erkenntnisse zutage fördern. Unsere begrenzte Untersuchung ist für unsere Zwecke dennoch ausreichend.

\section{B ÜBERSICHT DER BELEGE DER VERBINDUNG DES AUSDRUCKS „AM TAG“ MIT EINEM VERB IM STATUS CONSTRUCTUS}

Im Folgenden werden die 31 verschiedenen Belegstellen dargestellt, erst die Textangabe, dann das hebräische ,am Tag“ mit Verb im status constructus (mit und ohne Pronominalsuffix). Es folgt die wörtliche Übersetzung mit einer Verständnishilfe für den Leser in Klammern, danach die Funktionsbezeichnung als ,punktuell“, „zukünftig“ (mit zwei Möglichkeiten) und „,vergangen“. Schließlich betrachten wir die Stammformation des verwendeten hebräischen Verbs.

\begin{tabular}{|c|c|c|c|c|}
\hline Text $^{15}$ & Hebräisch & (wörtliche) Übersetzung & Funktion & Inf.cs. \\
\hline Gen 2,4 & בְּיוֹטם עִשֶׁוֹת & $\begin{array}{l}\text { am Tag des Machens (von Erde } \\
\text { und Himmel durch JHWH) }\end{array}$ & vergangen & qal \\
\hline Gen 2,17 & 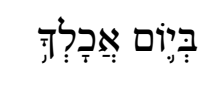 & $\begin{array}{l}\text { am Tag deines Essens (von dem } \\
\text { Baum sollst du Sterben) }\end{array}$ & $\begin{array}{l}\text { zukünftig } \\
\text { (offen) }\end{array}$ & qal \\
\hline Gen 3,5 & 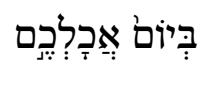 & $\begin{array}{l}\text { am Tag eures Essens } \\
\text { (von dem Baum) }\end{array}$ & $\begin{array}{l}\text { zukünftig } \\
\text { (offen) }\end{array}$ & qal \\
\hline Gen 5,1 & 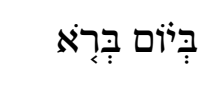 & $\begin{array}{l}\text { am Tag des Erschaffens von } \\
\text { (Adam) }\end{array}$ & vergangen & qal \\
\hline
\end{tabular}

\footnotetext{
13 Vgl. Brin, Concept, 55; Hodge, Revisiting, 79-80.

14 Dies schließt auch die entferntere Parallele Lev 14,57 von der Untersuchung aus, in welcher sich für Jenni, ,jōm“, 1: 711 der Begriff ,am weitesten [..] von der Bed. ,Tag““ entfernt, vgl. Bergman, „yôm“, 3: 568: „völlig abgeblaßt". Zu unterscheiden ist hier jedoch ein gnomischer Gebrauch des ,heute“ mit Bezug auf konkrete Tage in der Zukunft von der Ausdehnung eines bestimmten Zeitraumes auf unbestimmte Zeit, vgl. Simon DeVries, Yesterday, Today, and Tomorrw: Time and History in the Old Testament (Grand Rapids: Eerdmans, 1975), 45. Dass sich ein Tag in der Zukunft des Öfteren wiederholen kann, bedeutet nicht, dass hier etwas anders gemeint ist, als ein 24-Stunden-Tag, vgl. Hodge, Revisiting, 79.

15 Belegstellen nach der hebr./dt. Bibel (engl. Übersetzung: Lev 6,13 = 6,20; Num 30,6-15 = 30,5-14).
} 


\begin{tabular}{|c|c|c|c|c|}
\hline $\begin{array}{l}\boldsymbol{T e x t}^{15} \\
\text { Gen 5,2 }\end{array}$ & 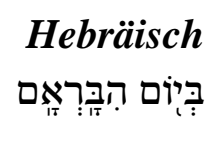 & 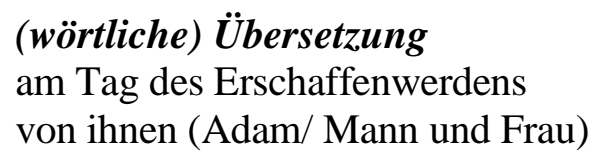 & $\begin{array}{l}\text { Funktion } \\
\text { vergangen }\end{array}$ & $\begin{array}{l}\text { Inf.cs. } \\
\text { nif'al }\end{array}$ \\
\hline Gen 21,8 & 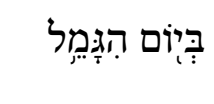 & $\begin{array}{l}\text { am Tag des Entwöhntwerdens } \\
\text { (von Isaak) }\end{array}$ & punktuell & nif'al \\
\hline Ex 6,28 & 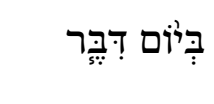 & $\begin{array}{l}\text { am Tag des Sprechens (durch } \\
\text { JHWH zu Mose im Land Ägypten) }\end{array}$ & vergangen & pi'el \\
\hline Ex 10,28 & 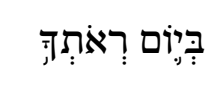 & $\begin{array}{l}\text { am Tag deines Sehens (meines } \\
\text { Angesichts wirst Du sterben) }\end{array}$ & $\begin{array}{l}\text { zukünftig } \\
\text { (offen) }\end{array}$ & qal \\
\hline Ex 32,34 & 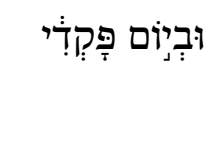 & $\begin{array}{l}\text { und am Tag meines Heimsuchens } \\
\text { (werde ich ihre Sünde an Ihnen } \\
\text { heimsuchen) }\end{array}$ & $\begin{array}{l}\text { zukünftig } \\
\text { (offen) }\end{array}$ & qal \\
\hline Lev 6,13 & 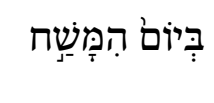 & $\begin{array}{l}\text { am Tag des Gesalbtwerdens (von } \\
\text { Aaron) }\end{array}$ & vergangen & nif'al \\
\hline Lev 7,16 & 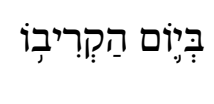 & $\begin{array}{l}\text { am Tag seines Darbringens (seines } \\
\text { Opfers) }\end{array}$ & $\begin{array}{l}\text { zukünftig } \\
\text { (sicher) }\end{array}$ & hif'il \\
\hline Lev 7,35 & בְּיוֹם הִקְּרִיב & $\begin{array}{l}\text { am Tag des Herzutretens (von } \\
\text { Aaron und seiner Söhne) }\end{array}$ & $\begin{array}{l}\text { zukünftig } \\
\text { (offen) }\end{array}$ & hif'il \\
\hline Lev 7,36 & 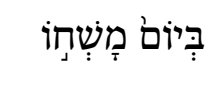 & $\begin{array}{l}\text { am Tag des Salbens durch ihn (von } \\
\text { Aaron und seiner Söhne) }\end{array}$ & $\begin{array}{l}\text { zukünftig } \\
\text { (offen) }\end{array}$ & qal \\
\hline Lev 7,38 & בְּיוֹס צַוּתוֹ & $\begin{array}{l}\text { am Tag des Befehlens durch ihn } \\
\text { (von den Söhnen Israel für die } \\
\text { Darbringung ihres Opfers) }\end{array}$ & vergangen & pi`el \\
\hline Lev 13,14 & וּבְיוֹם הֵרֶאוֹת & $\begin{array}{l}\text { und am Tag des Gesehenwerdens } \\
\text { (des wilden Fleisches) }\end{array}$ & $\begin{array}{l}\text { zukünftig } \\
\text { (sicher) }\end{array}$ & nif'al \\
\hline Lev 23,12 & 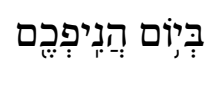 & am Tag des Bewegens (der Garbe) & $\begin{array}{l}\text { zukünftig } \\
\text { (sicher) }\end{array}$ & hif'il \\
\hline Num 3,1 & 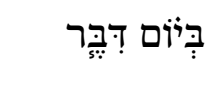 & $\begin{array}{l}\text { am Tag des Sprechens (JHWHs zu } \\
\text { Mose auf dem Berg Sinai) }\end{array}$ & vergangen & $\mathrm{pi}^{6} \mathrm{el}$ \\
\hline Num 3,13 & 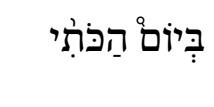 & $\begin{array}{l}\text { am Tag meines Schlagens (aller } \\
\text { Erstgeburt in Ägypten) }\end{array}$ & vergangen & hif'il \\
\hline Num 6,13 & בְּיוֹסם מְלאת & $\begin{array}{l}\text { am Tag der Erfüllung (der } \\
\text { Nasoräerschaft) }\end{array}$ & $\begin{array}{l}\text { zukünftig } \\
\text { (sicher) }\end{array}$ & qal \\
\hline Num 7,1 & בְּיוֹםْ כַּלוֹת & $\begin{array}{l}\text { am Tag der Vollendung (der } \\
\text { Wohnung durch Mose) }\end{array}$ & vergangen & $\mathrm{pi}^{6} \mathrm{el}$ \\
\hline Num 7,10 & 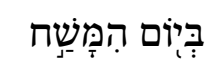 & am Tag der Salbung (des Altars) & vergangen & nif'al \\
\hline Num 7,84 & בְּיוֹם הִפְּשָׁח & am Tag der Salbung (des Altars) & vergangen & nif'al \\
\hline Num 8,17 & בִּיוֹטם הַכּתִת & $\begin{array}{l}\text { am Tag meines Schlagens (aller } \\
\text { Erstgeburt in Ägypten) }\end{array}$ & vergangen & hif'il \\
\hline Num 9,15 & וּבְיוֹם הִִָקים & $\begin{array}{l}\text { und am Tag der Aufrichtung } \\
\text { (bedeckte die Wolke die } \\
\text { Wohnung) }\end{array}$ & vergangen & hif‘il \\
\hline Num 30,6 & בְּיוֹס שָׁמְעוֹ & $\begin{array}{l}\text { am Tag des Hörens (ihrer } \\
\text { Gelübde) }\end{array}$ & $\begin{array}{l}\text { zukünftig } \\
\text { (sicher) }\end{array}$ & qal \\
\hline Num 30,8 & 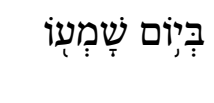 & $\begin{array}{l}\text { am Tag des Hörens (ihrer } \\
\text { Gelübde) }\end{array}$ & $\begin{array}{l}\text { zukünftig } \\
\text { (sicher) }\end{array}$ & qal \\
\hline
\end{tabular}


86 Riecker and Koorevaar, “Bedeutung,” OTE 33/1 (2020): 80-106

\begin{tabular}{|c|c|c|c|c|}
\hline $\begin{array}{l}\text { Text }^{15} \\
\text { Num } 30,9\end{array}$ & $\begin{array}{r}\text { Hebräisch } \\
\text { בְּיוֹם שְממעע }\end{array}$ & $\begin{array}{l}\text { (wörtliche) Übersetzung } \\
\text { am Tag des Hörens (seiner Frau) }\end{array}$ & $\begin{array}{l}\text { Funktion } \\
\text { zukünftig } \\
\text { (sicher) }\end{array}$ & $\begin{array}{l}\text { Inf.cs. } \\
\text { qal }\end{array}$ \\
\hline Num 30,13 & 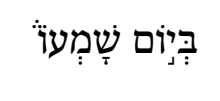 & $\begin{array}{l}\text { am Tag seines Hörens (von allem, } \\
\text { was über ihre Lippen kam) }\end{array}$ & $\begin{array}{l}\text { zukünftig } \\
\text { (sicher) }\end{array}$ & qal \\
\hline Num 30,15 & 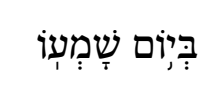 & $\begin{array}{l}\text { am Tag seines Hörens (Gelübde } \\
\text { seiner Frau) }\end{array}$ & $\begin{array}{l}\text { zukünftig } \\
\text { (sicher) }\end{array}$ & qal \\
\hline Dtn 4,15 & 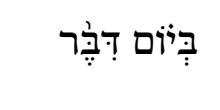 & $\begin{array}{l}\text { am Tag seines Redens (JHWH zu } \\
\text { Israel am Horeb) }\end{array}$ & vergangen & pi ${ }^{i} \mathrm{el}$ \\
\hline Dtn 21,16 & בְּיוֹם הַנְחִילִּוֹ & $\begin{array}{l}\text { am Tag des Besitzens (seiner } \\
\text { Söhne) }\end{array}$ & $\begin{array}{l}\text { zukünftig } \\
\text { (sicher) }\end{array}$ & hif' $\mathrm{il}$ \\
\hline
\end{tabular}

\section{KATEGORISIERUNG UND EXEGETISCHE BEOBACHTUNGEN IM PENTATEUCH}

Wenn man die Funktion der Vorkommen des Ausdrucks miteinander vergleicht, ergeben sich drei Möglichkeiten: Der Ausdruck kann auf den momentanen Zeitpunkt hinweisen (,punktuell“), in die Zukunft (,zukünftig“) oder in die Vergangenheit (,vergangen"). Bei einem Bezug auf die Zukunft gibt es wiederum zwei Möglichkeiten, die sich unterscheiden lassen: Die Zukunft bleibt „offen“ (abhängig von menschlichem Verhalten), die Zukunft liegt fest (,sicher“, mit einer Ordnung verbunden).

In jedem Text ist nun zu untersuchen, wie konkret sich die Aussage auf einen Tag bezieht oder nicht. Da sich Gen 2,4 auf die Vergangenheit bezieht, ist für unsere Diskussion diese dritte Kategorie von besonderer Bedeutung.

\section{1 „Am Tag“ mit punktuellem Bezug}

Wird ,am Tag“" punktuell auf die Gegenwart bezogen, bezieht sich der Ausdruck auf einen Tag, welcher in der gesellschaftlichen Tradition eine besondere Rolle spielt.

Gen 21,8. Der Tag, an welchem Isaak entwöhnt (von der Mutterbrust genommen) wurde. Isaak wurde entwöhnt, daraufhin wurde ein Festmahl gehalten. Dies war ein konkreter Tag. Es gibt einen Bezugspunkt für einen gesellschaftlich üblichen Brauch.

\section{2 „Am Tag“ bezogen auf die Zukunft}

Der Tag als Möglichkeit in der Zukunft

Wird „am Tag“ als offene Möglichkeit auf die Zukunft bezogen, ist damit im Pentateuch eine Drohung verbunden. An diesem Tag kann etwas Schlimmes geschehen, es muss nicht geschehen. Dies hängt von dem Verhalten einer Person oder Gruppe ab und ob eine gezogene Grenze überschritten wird oder nicht. Diese Formulierungen haben einen kasuistischen Charakter. 
Gen 2,17. Der Tag, an welchem der Mensch (Suffix 3. Pers. m. sg.) vom Baum der Erkenntnis von Gut und Böse isst, wird er sterben. Der Mensch darf von allen anderen Bäumen im Garten Eden essen, nur nicht von diesem einen. Der Mensch isst davon an einem konkreten Tag ohne Datum $(3,6)$, an welchem das Todesurteil ausgesprochen wird (3,17-19), vollzogen in seinem 930. Lebensjahr $(5,5)$.

Gen 3,5. Der Tag, an dem die Frau und ihr Mann (Suffix 2. Pers. m. pl.) von dem Baum der Erkenntnis von Gut und Böse essen, werden sie nicht sterben. Dies ist die Antithese zu 2,17, formuliert durch die Schlange (3,4-5).

Ex 10,28. Der Tag, an dem Mose das Angesicht des Pharaos sieht, soll er sterben. Er darf nicht mehr zum Pharao gehen, tut er es doch, wird er an jenen Tag getötet werden. Mose sagt, er werde tatsächlich das Angesicht des Pharaos nicht mehr sehen $(10,29) .{ }^{16}$

Ex 32,34. Der Tag der Heimsuchung Gottes, da wird er ihre Sünde heimsuchen. Nach dem Abzug vom Sinai werden allerlei Übertretungen berichtet, die Gott bestraft, wodurch viele Israeliten in einem bestimmten Augenblick umkommen (z. B. Num 11,33-34; 14,36-37; 16,31-36; 16,44-50; 21,4-9; 25,1-11). Das geschieht jeweils an einem konkreten Tag. Die erste Generation darf den Sinai wohl verlassen, erreicht Kanaan jedoch nicht. Der Aufstand in Num 13-14 ist der Tropfen, der das Fass zum Überlaufen bringt. Die Todesstrafe wird ausgesprochen (Num 14,20-23.28-29.31-32), die Verurteilten sterben unterwegs in der Wüste $(26,63-65 ; 27,3)$.

Der Tag als zukünftige Aufgabe oder zukünftige Ordnung

Wird ,am Tag“ auf einen festgelegten Tag in der Zukunft bezogen, geht es um einen zukünftigen Tag, an dem eine bestimmte Handlung vollzogen oder ein Auftrag ausgeführt wird, oft in Verbindung mit einer wiederkehrenden Ordnung.

Lev 6,13. Der Tag der Salbung von Aaron, seiner Söhne und späteren Priester. Im Falle Aarons hat dieser konkrete Tag im Pentateuch sogar ein explizit genanntes Datum, und zwar der achte Tag des ersten Monats des (zweiten) Jahres des Auszugs aus Ägypten (Lev 9,1.7.15).

Lev 7,16. Der Tag der Darbringung des Schlachtopfers als Gelöbnisopfer oder freiwilliges Opfer durch einen Israeliten. Dies ist jeweils ein konkreter Tag im Rahmen einer allgemeinen Ordnung.

16 Ex 12,31 zeigt, dass Mose das Angesicht des Pharao noch einmal straffrei gesehen hat. Die Initiative geht allerdings nicht von Mose aus sondern vom Pharao. Der Pharao wollte Mose sehen. 
88 Riecker and Koorevaar, “Bedeutung,” OTE 33/1 (2020): 80-106

Lev 7,35. Der Tag, an dem Aaron und seine Söhne herzutraten, um als Priester zu dienen. Das geschieht ebenfalls an dem betreffenden achten Tag des ersten Monats (vgl. 6,13).

Lev 7,36. Der Tag der Salbung Aarons und seiner Söhne. Dies geschieht ebenfalls am betreffenden achten Tag im ersten Monat.

Lev 13,14. Der Tag, an welchem lebendiges (wildes) Fleisch bei einem mit Hautausschlag (Aussatz) festgestellt wird. Ab dem Tag des Befundes ist die betreffende Person unrein. Dies ist jeweils ein konkreter Tag im Rahmen einer allgemeinen Ordnung.

Lev 23,12. Der Tag, an dem die (Erstlings-)Garbe geschwungen wird. Das Schwingen der Garbe beginnt am Tag nach dem (ersten) Sabbat des Festes der ungesäuerten Brote, also an einem Sonntag. Es handelt sich um den Sonntag nach dem Sabbat nach dem 15. des ersten Monats. Weil dies immer auf einen Sonntag fällt, variiert das Datum im Monatskalender von Jahr zu Jahr. Es ist allerdings immer ein konkreter Tag.

Num 6,13. Der Tag, an dem die Zeit der Enthaltung erfüllt ist. Die Länge des Zeitraums kann vom Nasiräer selber festgelegt werden. Dieser Tag kann auf jeden Tag des Jahres fallen, ist jedoch konkret und innerhalb einer allgemeinen Ordnung festgelegt.

Num 30,6. Der Tag, an dem der Vater vom Gelübde seiner Tochter hört. Eine Frau legt ein Gelübde ab, und ihr Vater erfährt davon. Das kann jeder Tag des Jahres sein, der Tag ist für den Mann konkret und entscheidend hinsichtlich seiner Verantwortung im Rahmen einer allgemeinen Ordnung.

Num 30,8. Der Tag, an dem ein Mann vom Gelübde seiner Frau erfährt. Siehe Num 30,6.

Num 30,9. Der Tag, an dem ein Mann vom Gelübde seiner Frau erfährt. Siehe Num 30,6.

Num 30,13. Der Tag, an dem ein Mann vom Gelübde seiner Frau erfährt. Siehe Num 30,6.

Num 30,15. Der Tag, an dem ein Mann vom Gelübde seiner Frau erfährt. Siehe Num 30,6.

Dtn 21,16. Der Tag, an dem jemand sein Erbe regelt, mündlich oder schriftlich. Die Regelung des Nachlasses kann vor dem Tod einer Person geschehen oder selbst während des Sterbens. Dieser Tag ist unbestimmt, aber wird im Rahmen einer allgemeinen Ordnung im Nachhinein für den oder die Erben sehr konkret. 
Etwas in der Vergangenheit ist so wichtig, dass sich der Verfasser darauf zurückbezieht. In den meisten Fällen steht der Ausdruck am Anfang einer neuen Perikope, wobei der Tag den Ausgangspunkt der weiteren Entwicklung bildet, für welche jener Tag den entscheidenden Anstoß gegeben hat.

Gen 5,1. Der Tag, an dem Gott den Menschen, Adam, schuf. Der benannte Tag in der Vergangenheit wird in der zweiten Satzhälfte durch die Angabe spezifiziert, dass Gott ,ihn“ (א אi 'ōtô, 3. Pers. m. sg., 5,1b) an diesem Tag nach seinem Bilde erschafft. Dies geschieht am sechsten Tag des Schöpfungsberichts (1,27a). In 5,1a findet sich zum zweiten Mal im Buch Genesis die ToledotFormel, diesmal verbunden mit Adam. Was ist aus der Schöpfung von Adam hervorgegangen? Von 5,3 an erscheint eine Reihe aufeinanderfolgender Namen von Adam bis Noah und seinen Söhnen (5,3-32). Vers 5,1 gleicht in seiner Struktur $2,4 .{ }^{17}$ Es beginnt mit der Toledot-Formel, welcher der Ausdruck , an dem Tag" folgt. Damit liegt diese zweite Folge von Toledot und ,an dem Tag" in der Verlängerung der ersten Folge von Toledot und ,an dem Tag“ $(2,4)$ im Schöpfungsbericht von 1,1-2,3.

Gen 5,2. Der Tag, an dem sie geschaffen wurden (Suffix 3. Pers. m. pl.). Vor dieser abschließenden Terminierung werden über den Tag drei Informationen mitgeteilt: Männlich und weiblich erschafft Gott „sie“ (Suffix),

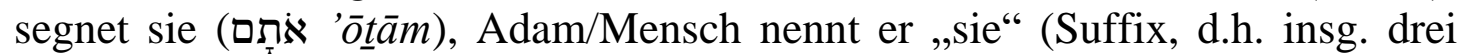
Suffixe 3. Pers. m. pl.). Das geschah am sechsten Tag des Schöpfungsberichts (1,27b-28). ${ }^{18}$ Auffallend ist, dass gleich zweimal hintereinander in 5,1 und 5,2 zurückgegriffen wird auf denselben Tag der Schöpfung des Menschen.

Ex 6,28. Der Tag, an dem JHWH in Ägypten zu Mose sprach. Der knappe Inhalt der Rede in 6,29-7,5 deutet auf einen kurzen Zeitabschnitt innerhalb eines konkreten Tages. Der Abschnitt 6,28-30 ähnelt inhaltlich sehr stark dem, was in 6,10-12 (bzw. 6,10-13) berichtet wird. Gott spricht zu Mose, nachdem Mose zum ersten Mal beim Pharao gewesen ist und durch seinen Auftritt die Sache eher verschlechtert als verbessert hat $(5,22-6,13)$. Was in $6,10-12$ berichtet wird, ist an einem konkreten Tag geschehen, den wir nicht mehr genau bestimmen können.

Lev 7,38. Der Tag, an dem JHWH den Israeliten gebot, ihre Opfergaben in der Wüste Sinai darzubringen. Diese Zeitangabe findet sich am Ende der Schlussformel des Gesetzes über die unterschiedlichen Opfer (7,37-38). JHWH gibt Mose das Opfergesetz bereits zuvor auf dem Berg Sinai (V.38a), ,an dem

17 Vgl. die tabellarischen Gegenüberstellungen bei Martin Arneth, Durch Adams Fall ist ganz verderbt ...: Studien zur Entstehung der alttestamentlichen Urgeschichte (Göttingen: Vandenhoeck \& Ruprecht, 2007), 132 und Hodge, Revisiting, 88.

18 Die Namensgebung Adams steht nicht direkt in 1,27-29. Es ist möglich, dies indirekt im Selbstanruf Gottes zu sehen, Adam/den Menschen zu schaffen $(1,26)$. 
Tag“, an dem er den Israeliten befiehlt, ihre Opfer in der Wüste Sinai darzubringen (V.38b). In diesem Vers wird zwischen Berg Sinai und Wüste Sinai unterschieden. Der Ausdruck, ,an dem Tag“ steht in Verbindung mit der Wüste Sinai.

Der Ausdruck ,an dem Tag“ am Abschluss des Opfergesetzes ist mit dem Gebot verbunden, die in Lev 1-7 beschriebenen Opfer in der Wüste Sinai darzubringen. Das geschieht am ersten Tag des ersten Monats des (zweiten) Jahres des Auszuges (vgl. Lev 1,1 mit Ex 40,1.34-35; vgl. Lev 8,1; 9,1).

Die Einsetzung der Opfer selber geschieht jedoch bereits etwa acht Monate vorher auf dem Berg Sinai (Ex 29). Ein genaues Datum für dieses Ereignis ist nicht oder nur schwer feststellbar, aber es liegt wohl im Zeitraum der ersten vierzig Tage des Mose auf dem Berg Sinai.

Num 3,1. Der Tag, an dem JHWH auf dem Berg Sinai mit Mose redete. Obwohl die Zahl im Kontext nicht angedeutet wird, erscheint es oberflächlich betrachtet nicht abwegig, den „Tag“ in Num 3,1 mit Umberto Cassuto auf die vierzig Tage und vierzig Nächte im Buch Exodus zu beziehen. ${ }^{19}$ Exodus berichtet über zwei Perioden von vierzig Tagen und Nächten von Mose auf dem Sinai, vor und nach dem Bundesbruch (Ex 24,18; 34,28). ${ }^{20}$ Über die ersten vierzig Tage wird ausführlich informiert. Gott redet zu Mose $(25,1)$ und gibt Gesetze über den Bau der Stiftshütte (Ex 25-27; 30-32) und das aaronitische Priestertum (Ex 28-29). Eine thematische Verbindung zwischen Num 3 und Ex 25-32 ist unübersehbar, denn auch in Num 3-4 geht es um das Priestertum und die Stiftshütte, untrennbar verbunden mit den Leviten. Die Aufgabe der Leviten ist es, die Stiftshütte (einschließlich der Geräte) abzubauen, zu transportieren und wieder aufzubauen, und zwar unter der Leitung Aarons und seiner Söhne. ${ }^{21}$

19 Umberto Cassuto, A Commentary on the Book of Genesis. Part I. From Adam to Noah. Genesis I - VI 8 (Jerusalem: Magnes, 1998), 99.

20 In jener Zeit geschehen folgende Dinge: Mose besteigt zum ersten Mal den Berg und kehrt zurück zur Vorbereitung des Bundesschlusses; das Hören der Zehn Worte; das Hören und das Überbringen der Vorschriften des Bundesbuches; der Bundesschluss; die ersten vierzig Tage des Mose auf dem Berg zum Empfang der Vorschriften für den Bau des Heiligtums und die Einsetzung des Priestertums mit dem Empfang der zwei Steintafeln mit den Zehn Worten; der Bundesbruch mit dem goldenen Stierkalb und die zweiten vierzig Tage des Mose auf dem Berg, um die Versöhnung herzustellen. Vgl. Gregory Chirichigno, „The Narrative Structure of Exod 19-24“, Bib 68 (1987): 457-479; Daniel C. Arichea, „Note. The ups and downs of Moses: locating Moses in Exodus 19-33“, BiTr 40 (1989): 244-246 und Thomas B. Dozeman, „Spatial Form in Exod 19:1-8a and in the Larger Sinai Narrative“, Semeia 46 (1989): 95-97.

21 Num 3,25-26.31-32.36-37; 4,4-16.24-28.31-33. 
Über den zweiten Zeitraum von vierzig Tagen ist bekannt, dass Gott dem Mose den Inhalt der zweiten Hälfte des Bundesbuchs neu mitteilt (Ex 34,10-26; vgl. 23,14-33), welche Mose niederschreibt. Auch die Zehn Worte werden erneuert (34,1.28). Für die Mitteilung des Inhalts dieser wenigen Verse wird viel weniger als einen Tag notwendig gewesen sein. Es wird spätestens hier sehr deutlich, dass die Menge der vierzig Tage nicht für den Empfang des Gesetzes notwendig ist. Literarisch wird diese Zahl mit Symbolwert vielmehr mit zwei anderen Dingen in Verbindung gebracht: (1) Mose ist in der Gegenwart JHWHs und überlebt das Menschenunmögliche. (2) Mose fastet und überlebt ohne Brot und Wasser (34,27-28), versorgt durch Gottes Herrlichkeit.

Diese Verbindung wird noch deutlicher beim ersten Zeitraum betont: Mose geht mitten hinein in die Wolke der Herrlichkeit JHWHs (23,15-18). Die vierzig Tage dienen der Begegnung mit Gott und der Heiligung, begleitet durch Fasten - eine Vorbereitungszeit. Erst ,am Ende der vierzig Tage und vierzig Nächte“ erhält Mose die Bundestafeln (Dtn 9,9-11). ${ }^{22}$ Wenn Ex 24,16 nicht zeitlich vor, sondern umschreibend parallel $z u 25,1$ formuliert, würde dies darauf hindeuten, dass die Gebote Ex 25-32 nicht am vierzigsten, sondern am siebten Tag gegeben wurden. ${ }^{23}$

Eine genauere Untersuchung macht deutlich, dass sich Num 3,1b nicht auf die Leviten im Allgemeinen, sondern auf die Todelot von Aaron und Mose bezieht (V.1a), nämlich „Nadab und Abihu, Eleasar und Itamar“, die ,Söhne Aarons“(V.2), ein wörtlicher Bezug auf Ex 28,1. Diese kurze Toledot wurde an einem konkreten Tag auf dem Berg mitgeteilt. ${ }^{24}$

Eine inhaltliche und formale Verwandtschaft von den „Aaron und Mose“Stellen Num 3,1 und Ex 6,28 lässt sich kaum bestreiten. Ex 6,28 schließt eine aaronitischen Genealogie (und den Abschnitt 6,13-27) ab, Num 3,1 leitet eine aaronitische Genealogie (und den Abschnitt 3,2-4,49) ein. In beiden Fällen findet sich eine Doppelung („Aaron und Mose“/ „Mose und Aaron“ in Ex 6,26-27; „die Namen der Söhne Aarons“ in Num 3,2-3). Gemeinsam mit Dtn 4,15 sind es die

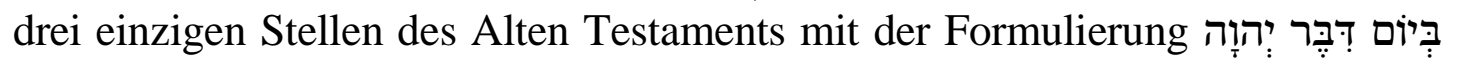
bəjôm dibber JHWH, am Tag, als JHWH sprach“. In allen drei Fällen unterstreicht die Ortsbezeichnung (Ägypten, Sinai, Horeb) die Wirklichkeit des Tages.

Num 3,13. Der Tag, an dem JHWH jede Erstgeburt im Land Ägypten schlug. Diese Formulierung bildet die Einleitung zu der Ordnung, die Leviten

22 Hodge, Revisiting, 75-76.

23 Wenn Ex 24,15-17 das folgende zusammenfasst, reduziert sich der Zeitraum des Redens Gottes auf maximal 34 Tage, vgl. zur Diskussion Cornelis Houtman, Exodus. Volume 3: Chapters 20 - 40 (Kampen: Kok, 2000), 305. Zudem zeigt Gott dem Mose während dieser Zeit ein Modell der Stiftshütte $(25,9)$.

24 Die zitierte Liste endet mit Num 3,2. Num 3,3-4 erläutert nur noch mit Hilfe weiterer Informationen (Ex 28,41; Lev 10,1-2). 
vor JHWH zu zählen und anstelle der Erstgeborenen von Israel zu weihen (Num 3,11-4,49). Jenes Gericht wird in der Nacht des 14. Aviv vollzogen (Ex 12,6.12.18.29-30). Siehe auch Num 8,17.

Num 7,1. Der Tag, an dem Mose die Wohnung fertig aufgerichtet hatte. Diese Formulierung bildet die Einleitung zur Darbringung der Opfergaben der Fürsten Israels zum Transport der Wohnung Gottes und der Weihegeschenke für den Altar (7,1-88). Das geschieht am ersten Tag des ersten Monats des zweiten Jahres des Auszuges (Ex 40,1.17). Siehe auch Num 9,15, wo derselbe Ausdruck hāqîm 'et-hammiškān ,die Wohnung aufrichten“ vorkommt.

Num 7,10. Der Tag, an dem der Altar gesalbt wurde. Diese Formulierung steht im Rahmen der Darbringung der Opfergaben der Fürsten Israels zum Transport der Wohnung Gottes und der Weihegeschenke für den Altar (7,1-88). Das geschieht am ersten Tag des ersten Monats des zweiten Jahres des Auszuges (Ex 40,1.10). Siehe auch 7,84.

Num 7,84. Der Tag, an dem der Altar gesalbt wurde. Siehe 7,10. ${ }^{25}$

Num 8,17. Der Tag, an dem JHWH jede Erstgeburt im Land Ägypten schlug. Diese Formulierung steht im Rahmen der Weihe der Leviten (8,5-22.2326). Das Gericht wird in der Nacht des 14. Aviv vollzogen (Ex 12,6.12.18.2930). Siehe auch Num 3,13, wo derselbe Ausdruck vorkommt.

Num 9,15. Der Tag, an dem JHWHs Wohnung aufgerichtet wurde und die Wolke die Wohnung bedeckte. Diese Formulierung bildet die Einleitung zur

25 Num 7,84 ist für Cassuto, Genesis I, 99 neben Num 3,1 der zweite von zwei Belegen aus der Tora für die Annahme, dass der Ausdruck in Gen 2,4 „does not signify specifically a day of twelve or twenty-four hours“. Die Begründung lautet: „the offering of the sacrifices of the princes lasted twelve days". V. 88 macht jedoch deutlich, dass die Salbung des Altars in Num 7,1 abgeschlossen ist und sich Überführung der Opfergaben an diesen Tag anschließt. Der Ausdruck דִ̣ bəjôm mit Infinitiv constructus wird in diesem Kapitel durchgehend auf den konkreten Tag der Salbung bezogen (V.1.10.84), מכּ bajjôm mit Ordinalzahl (,am ersten Tag“ ... ,,am zehnten Tag“, V.12.18.24.30.36.42.48.54.60.66), bzw. bọ bojôm mit Ordinalzahl (,am elften/zwölften Tag“, V.72.78) auf die Tage der Überführung. Die Ordinalzahlen sind hier also nicht Indikator zur Unterscheidung zwischen wörtlichem und nichtwörtlichem „Tag“, gegen Robert V. McCabe, „A Critique of the Framework Interpretation of the Creation Week", in Coming to Grips with Genesis. Biblical Authority and the Age of the Earth (Hg. Tarry Mortenson und Thane H. Ury; Green Forrest: Master, 2008), 228. Vgl. Hodge, Revisiting, 76-77. Neben diesen beiden Belegen nennt Cassuto 2. Sam 22,1 = Ps 18,1: „obviously it was not in one day that the Lord delivered David from all his enemies“ (100). Auch dieses Argument kann schwerlich überzeugen: David schreibt nicht kontinuierlich während der Zeit der Kämpfe, sondern an einem konkreten Tag, an dem der letzte Feind besiegt ist und David seinem Gott rückblickend für die erfolgte Rettung danken kann. 
Beschreibung der Funktionsweise der Wolke und Feuersäule bei der Wohnung während der Reise. Die Wohnung wird aufgerichtet am ersten Tag des ersten Monats des zweiten Jahres des Auszuges (Ex 40,1.17.34-35). Siehe auch Num 7,1.

Dtn 4,15. Der Tag, an dem JHWH am Horeb aus dem Feuer heraus zu Israel sprach. Das Reden von JHWH aus dem Feuer heraus steht am Beginn des Aufenthalts von Israel am Sinai in Ex 19,16-20 und 20,18. Das Ereignis findet statt ,am dritten Tag“ $(19,16)$, also an einem ganz konkreten Tag.

\section{VORLÄUFIGE SCHLUSSFOLGERUNGEN}

In fast allen behandelten Fällen handelt es sich um einen konkreten Tag, in der Regel sogar genau datiert. In einem Fall könnte es sich bei ,am Tag“ um eine begrenzte Anzahl von Tagen handeln (Num 3,1: vierzig Tage beim Aufenthalt des Mose auf dem Berg Sinai in Ex 25-31). Hier ist der erste Tag dieser Anzahl der Anstoß und möglicherweise sogar der entscheidende Tag, um den es geht.

Es gibt einige Tage in der Vergangenheit, die so wichtig gewesen sein müssen, dass zweimal mit diesem Ausdruck auf sie Bezug genommen wird. Diese sind der Tag der Schöpfung des Menschen (Gen 5,1.2), der Tag, an dem JHWH die Erstgeburt von Ägypten schlägt (Num 3,13; 8,17), der Tag der Aufrichtung der Wohnung JHWHs (Num 7,1; 9,15) und der Tag, an dem der Altar gesalbt wird (Num 7,10.88). Auch auf das Reden JHWHs wird zweimal Bezug genommen, allerdings mit Variation: Im ersten Text spricht JHWH zu Mose auf dem Berg Sinai (Num 3,1), und im zweiten Text spricht er zu Israel auf dem Horeb (Dtn 4,15).

In drei Fällen steht der Ausdruck ,,an dem Tag““ in einem Vers, der mit der Toledot-Formel einsetzt:

$\begin{array}{ll}\text { Gen 2,4 } & \begin{array}{l}\text { die Toledot } \text { von } \\ \text { Himmel und Erde }\end{array} \\ \text { Gen 5,1 } & \begin{array}{l}\text { die Toledot } \text { von Adam } \\ \text { die Toledot } \text { von Aaron und } \\ \text { Num 3,1 } \\ \text { Mose }\end{array}\end{array}$

Wie die Parallelstellen Ex 6,28 und Dtn 4,15 (die beiden einzigen Stellen neben Num 3,1, welche den Tag mit einem Reden JHWHs verbinden, s.o.) weist Num 3,1 deutlich auf einen einzelnen konkreten Tag zurück.

Dies wiederum bestätigt den Eindruck, dass auch die beiden anderen Toledot mit ,am Tag“ Bezug nehmen auf einen einzelnen konkreten Tag im Schöpfungsbericht in Gen 1,1-2,3: Gen 2,4 mit עשהה sh ,machen“, qal, und Gen 
ברא ,erschaffen“, qal (vgl. darüber hinaus ohne Toledot Gen 5,2 ברא 5,1 mit ברא $b r$ ',erschaffen“, nif'al). ${ }^{26}$

Der Blick über die Tora hinaus zeigt, dass dem Verfasser zur Bezeichnung eines längeren Zeitraums die Möglichkeit offenstand, diese Konstruktion mit einem Plural zu bilden. Im gesamten hebräischen Kanon gibt es für diese

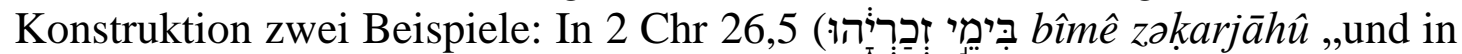
den Tagen Secharjahus“") geht es um eine unbekannte Zeit (Gegenwart), während welcher der König Usia unterwiesen wird und Gott sucht. Es können viele Jahre gewesen sein, der Chronist legt sich auf keinen genauen Zeitraum fest. Rut 1,1

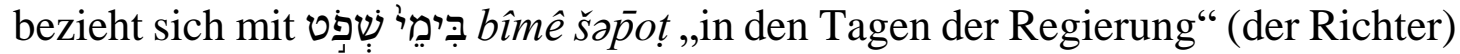
zurück in die Vergangenheit, eine Zeitspanne, die mehr als drei Jahrhunderte umfasst haben mag (vgl. Ri 11,26).

Diese Beobachtung unterstreicht, dass die seit Jahrhunderten kontinuierlich wiederholte Behauptung, der Ausdruck im Singular ziele auf eine lange Periode, durch Parallelen in der Tora nicht belegt werden kann.

\section{E GEN 2,4 ALS TAG IN DER VERGANGENHEIT - EXEGETISCHE ÜBERLEGUNGEN UND ERGEBNIS}

\section{Genesis 2,4b im Kontext von Genesis 1,1}

Es steht außer Frage, dass sich Genesis 2,4b mit der Aussage ,am Tag, als JHWH Gott Erde und Himmel machte“ auf den vorangehenden Schöpfungsbericht zurückbezieht (Gen 1,1-2,3). Die Frage ist jedoch, worauf genau? Bezieht sich die Aussage auf die gesamte Schöpfungsperiode, alle sechs Tage (1,1-31), dann wäre ,Tag“ zu verstehen im Sinne eines exakt abgegrenzten Zeitraums von sechs Tagen. Durch ihren wörtlichen Bezug greift die Aussage jedoch eindeutig direkt auf 1,1 zurück. Das bedeutet noch nicht, dass sich 2,4b auf den ersten Schöpfungstag zurückbezieht, welcher in 1,5 abgeschlossen wird. Denn der Beginn des ersten Schöpfungstages ist umstritten, d.h. die Frage, ob 1,1 bereits Teil der Beschreibung des Tages ist, oder in einer allgemeineren Form die Ereignisse des gesamten Kapitels umfasst.

In der Geschichte der Auslegung lassen sich mit James Barr drei verschiedene Vorschläge zum Verständnis von 1,1 unterscheiden:

26 Es gibt deutliche Hinweise im Text, die davor warnen, den Unterschied zwischen den Verben עשה br' „sh „schaffen“ überzubetonen. In Gen 5,1b findet sich „schaffen“, in 5,1b $\beta$ wird für dasselbe Geschehen das Verb „machen“ verwendet, vgl. Markus Witte, Die biblische Urgeschichte: Redaktions- und theologiegeschichtliche Beobachtungen zu Genesis 1,1 - 11,26 (Berlin: De Gruyter, 1998) 54, Anm. 7. 
1 Genesis 1,1 beschreibt eine erste Tat der Schöpfung, vor der Erschaffung von Licht in V.3.

2 Der Vers ist ein temporaler Nebensatz im Sinne von ,als Gott sich daran machte, Himmel und Erde zu erschaffen“, verbunden mit der Beschreibung des Chaos in V.2.

3 Der Vers ist eine Zusammenfassung des gesamten Schöpfungswerks, welchem dann eine detailliertere Darstellung des gesamten Schöpfungsprozesses über sieben Tage hin folgt. ${ }^{27}$

Ähnlich wie Barr ${ }^{28}$ machen auch andere Ausleger darauf aufmerksam, mit welchen syntaktischen Schwierigkeiten Möglichkeit 2 behaftet ist. Michaela Bauks redet in ihrer Monographie zu Genesis 1,1-3 von einem hypotaktischen Erklärungsmodell (unterschieden von einem parataktischen Modell) ${ }^{29}$ und zählt eine ganze Reihe grammatischer Einwände gegen diese Lösung auf ${ }^{30}$. Nach Walter Bührer ist der (bis heute beobachtbare) ${ }^{31}$ Hang zu einem hypotaktischen Modell in der kritischen Forschung dabei vor allem auf inhaltliche Gründe zurückzuführen: Da die klassische Idee einer creatio ex nihilo (Augustinus) untrennbar mit dem parataktischen Modell verbunden scheint, man diese jedoch als griechisch-philosophische Überfremdung der hebräischen Bibel ablehnt, ${ }^{32}$ nimmt man Zuflucht zu dem scheinbar geringeren Übel einer unwahrscheinlicheren Satzkonstruktion. ${ }^{33}$ Dieses Vorgehen erscheint

27 James Barr, „Was Everything that God Created Really Good? A Question in the First Verse of the Bible“, in God in the Fray. A Tribute to Walter Brueggemann (Hg. Tod Linafelt und Timothy K. Beal; Minneapolis: Fortress, 1998), 55. Vgl. dasselbe Schema bei Samuel D. Giere, A New Glimpse of Day One: Intertextuality, History of Interpretation, and Genesis 1.1-5 (Berlin: De Gruyter, 2009), 19, Anm. 12 mit weiteren Belegen, welcher ohne Kenntnis von Barr, Bauks, Weippert und Arneth für Möglichkeit 2 votiert.

28 Barr, „Everything“, 58, der sich jedoch noch stärker von Möglichkeit 1 distanziert.

29 Nach dem parataktischen Modell steht Gen 1,1 als eigenständiger Satz entweder als Überschrift (auch hier umfasst die Aussage die gesamte Schöpfung 1,1-31) oder als Beschreibung der ersten Schöpfungshandlung prima creatio. Letzteres klassisches Verständnis ordnet die Erschaffung des Himmels und der Erde dem ersten Tag zu. Hier lässt sich auch die Idee der creatio ex nihilo (Augustinus) einordnen, Michaela Bauks, Die Welt am Anfang: Zum Verhältnis von Vorwelt und Weltentstehung in Gen 1 und in der altorientalischen Literatur (Neukirchen-Vluyn: Neukirchener, 1997), 28-29, 69-86. 30 Bauks, Welt am Anfang, 71-73.

31 Vgl. Schüle, Urgeschichte, 32-33; ferner Arneth, Adams Fall, 25, Anm. 17; Manfred Weippert, „Schöpfung am Anfang oder Anfang der Schöpfung? Noch einmal zu Syntax und Semantik von Gen 1,1-3“, TZ 60 (2004): 14-15.

32 Bauks, Welt am Anfang, 63-64.

33 Walter Bührer, Am Anfang ...: Untersuchungen zur Textgenese und zur relativchronologischen Einordnung von Gen 1-3 (Göttingen: Vandenhoeck \& Ruprecht, 2014) 101. 
methodisch zumindest fragwürdig und nach Bührer und Bauks zudem unnötig, da auch Möglichkeit 3 im Rahmen des parataktischen Modells liegt und so deuten sie die ersten Worte der Genesis als Überschrift zu 1,1-31. ${ }^{34}$ Die Deutung als Überschrift macht jedoch aus erzählerischer Sicht kaum Sinn, da 1,2 formal betrachtet als erzählinitialer Satz so gut wie unmöglich ist. ${ }^{35}$ Das erfordert weitere wenig überzeugende Ad-hoc-Hypothesen zur Rettung des Modells, etwa die „Vorweltschilderung“ $(1,2)$ syntaktisch der Überschrift zuzuordnen (die damit aber keine Überschrift mehr wäre), gleichzeitig aber gegen die Syntax semantisch ,kontrastiv“ dem folgenden Vers 3.

So verständlich die Abneigung gegen ontologische Streitigkeiten im exegetischen Bereich auch sind - Barr hat noch einmal deutlich gezeigt, dass Möglichkeit 1 hellenistisches Denken keineswegs zwangsläufig voraussetzt, sondern lediglich den simplen Gedanke an eine Schöpfung und die allgemeinmenschliche Frage: ,Warum sollte es eine Welt geben?“36 Da der philosophische Einwand der genaueren Prüfung nicht standhalten kann, ist dieses mit Abstand früheste „klassische“ Modell für viele Exegeten von Wellhausen über Gunkel, Childs und Wenham bis heute bei einem natürlichen Verständnis die offensichtliche Lösung: ${ }^{37}$ Gen 1,1 muss als Teil des ersten Tages gedeutet werden, denn rein logisch betrachtet setzt die in Vers 2 auftauchende Erde den in Vers 1 beschriebenen Anfang/Grund dieser Erde unabdingbar voraus.

34 Bauks, Welt am Anfang, 86: „Überschrift- bzw. Mottovers-Charakter“, 3: „überschriftartig“, 5: „mottohaft“. Ähnlich Bührer, Am Anfang, 107 [ohne Hervorhebungen]: „Gen 1,1 als Überschrift und 1,2 als kontrastive Vorweltschilderung“" Weitere Belege bei O.H. Steck, Der Schöpfungsbericht der Priesterschrift. Studien zur literarkritischen und überlieferungsgeschichtlichen Problemantik von Genesis 1,1-2,4a (FRLANT 115; Göttingen 21981).

35 So selbst Bührer, Am Anfang, 110. Vgl. Bauks, Welt am Anfang, 141-146.

36 Barr, „Everything“, 65 zitiert Maurice F. Wiles, God's Action in the World. The Bampton Lectures for 1986 (London: SCM, 1986), 16: „Creation is creation out of nothing or it is nothing."

37 Während Julius Wellhausen, Prolegomena zur Geschichte Israels (6. Aufl.; Berlin: De Gruyter, 1927), 296 noch davon ausgeht, dass Gott in Gen 1,1 etwa erschafft, was in Gen 1,2 als Chaos beschrieben wird - „es ist ein merkwürdiger Gedanke“-, arbeitet Gunkel, Genesis, 102 mit literarischen und zeitlichen Umstellungen: 2,4a wird dem Abschnitt als Überschrift literarisch vorangesetzt. Die Schöpfungstat in 1,1 folgt der Schilderung des Chaos zeitlich. Es ist bemerkenswert, dass Gunkel trotz dieser aufwändigen Umstellungen die ihm bekannte temporale Lösung für unwahrscheinlicher erklärt. Gordon J. Wenham, Genesis 1-15 (Waco: Word, 1987), 1113 unterstreicht in seiner Untersuchung die Bedeutung der masoretischen Punktation, der Septuaginta und frühesten Übersetzungen und nennt zahlreiche weitere neuzeitliche Vertreter. Vgl. Brevard S. Childs, Myth and Reality in the Old Testament (London: SCM, 1960), 31-43. 
Welche Auswirkungen hat diese Diskussion nun auf das Verständnis von Genesis 2,4b? Wenn es sich bei Gen 1,1 nach diesem Verständnis um die erste und einleitende Schöpfungstat Gottes handelt (die Schöpfung von Himmel und Erde „im Rohbau“; Möglichkeit 1, parataktisch), dann bezieht sich Gen 2,4 eindeutig auf den ersten Tag, welcher in Gen 1,1-5 dargestellt wird.

\section{Genesis 2,4b im Kontext von Genesis 2,4a}

Ob es möglich oder legitim ist, 2,4b im Kontext von 2,4a zu lesen, wird in vielen Untersuchungen nicht an der syntaktischen Struktur des Verses, sondern an einem Gesamtkonzept von postulierten Quellen, Fragmenten und Ergänzungsschichten entschieden. Wenn beispielsweise Gen 2,4b-3,25 einem Jahwisten zugeordnet wird, der sein Werk einige Jahrhunderte vor einer in 1,12,4a lokalisierten Priesterschrift verfasst, wäre es chronologisch unmöglich, dass 2,4b an 2,4a anknüpft. Dieses klassische Modell der neueren Urkundenhypothese ist mittlerweile durch eine Menge an widerstreitenden Modellen ergänzt worden. Nicht selten wird hier die in Gen 2,4b beginnende Quelle oder Ergänzungsschicht (wie vor Wellhausen üblich) zeitlich nach Gen 2,4a datiert. ${ }^{38}$ Doch auch ungeachtet der zeitlichen Anordnung war es immer möglich, chronologisch bedingte Widersprüche durch die Annahme von Redaktoren und Nachträgen zu überwinden.

Die „Leidensgeschichte“ des zerteilten Verses geht zurück bis ins Ende des 18. Jh. ${ }^{39}$ Bis heute wird dabei immer wieder auch für eine Einheit von Genesis 2,4 als Teil der darauf folgenden Erzählung bzw. „bündig als Überleitung “40 argumentiert. Die gegenwärtige Vielfalt der Lösungsvorschläge

38 So Eckart Otto, „Die Paradieserzählung Genesis 2-3: Eine nachpriesterschriftliche Lehrerzählung in ihrem religionshistorischen Kontext", in ,, Jedes Ding hat seine Zeit ...": Studien zur israelitischen und altorientalischen Weisheit. Diethelm Michel zum 65. Geburtstag (Hg. Anja A. Diesel u.a.; Berlin: De Gruyter, 1996), 188: „Gen 2,4-3,24 setzt also durchgängig die Priesterschrift voraus und führt einen Dialog mit dem priesterschriftlichen Schöpfungsbericht"; Andreas Schüle, „Made in the "Image of God“: The Concepts of Divine Images in Gen 1-3“, ZAW 117 (2005): 1-20, insb. 3, Anm. 7. Vgl. Erhard Blum, „Von Gottesunmittelbarkeit zu Gottesähnlichkeit: Überlegungen zur theologischen Anthropologie der Paradieserzählung“, in Textgestalt und Komposition: Exegetische Beiträge zu Tora und Vordere Propheten (Hg. Wolfgang Oswald; Tübingen: Mohr, 2010), 6-9, kritisch zu Blenkinsopp, Otto und Spieckermann.

39 Zuletzt erneut verteidigt durch Alfred Marx, „La fin du récit sacerdotal de la création: Gn 2,4a ou Gn 2,3?“, VT 67 (2017): 581-588. Zur Geschichte vgl. Terje Stordalen, „Genesis 2,4. Restudying a locus classicus“, ZAW 104 (1992): 163166. Witte, Urgeschichte, 53-54. Otto, „Paradieserzählung“, 185-187.

40 Blum, ,Gottesunmittelbarkeit“, 7, Anm. 27; ähnlich Stordalen, „Genesis 2,4“, 163 177; Otto, „Paradieserzählung“, 185-187; Jack Collins, „Discourse Analysis and the Interpretation of Gen 2:4-7“, WTJ 61 (1999): 272; Gordon J. Wenham, „The Priority of 
macht deutlich, dass eine literarkritische Sichtweise nicht zwangsläufig zu einer Scheidung von 2,4a und 2,4b führen muss. Eine nicht unter „Systemzwang “41 stehende Analyse wird an der chiastischen Struktur des Verses entscheiden, ob hier eine oder mehrere Hände am Werk waren: ${ }^{42}$

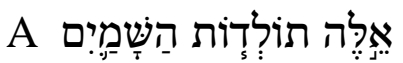

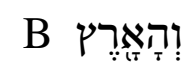

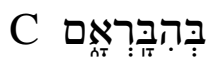

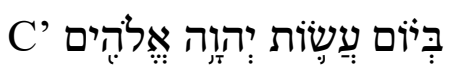

B' אוּרִ

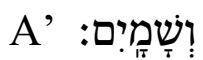

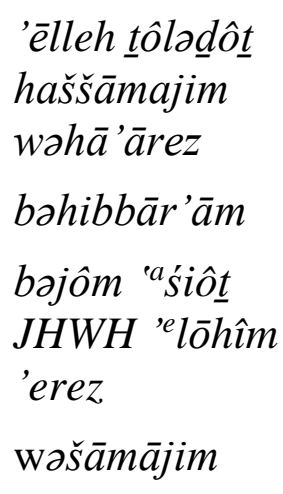

„dies sind die Toledot des Himmels“ ,und der Erde“

„als sie geschaffen wurden“"

,am Tag als JHWH Gott machte"

„Erde“"

,und Himmel“"

Seit Umberto Cassuto $^{43}$ ist immer wieder auf diesen Chiasmus hingewiesen worden als Argument für eine bewusste Gestaltung und Einheit des Verses. Gleichzeitig ist dieser aber auch stärkstes Argument gegen eine Einheitlichkeit von $2,4,{ }^{44}$ da sich der Wegfall der Determination im Chiasmus (,des Himmels und der Erde“ - „Erde und Himmel“) bei einem einzelnen Verfasser kaum erklären lässt. ${ }^{45}$ Die Abfolge „Erde und Himmel“" ist auch in

P“, VT 49 (1999): 252; Marc Vervenne, „Genesis 1,1-2,4: The Compositional Texture of the Priestly Ouverture to the Pentateuch", in Studies in the Book of Genesis: Literature, Redaction, History (Hg. André Wénin; Leuven: Peeters, 2001), 48, 69.

41 Fischer, Genesis 1-11, 178, Anm. 1.

42 Chiasmus nach Arneth, Adams Fall, 131.

43 Cassuto, Genesis I, 98-99, weitere Belege in Peter Weimar, „Struktur und Gestalt der priesterschriftlichen Schöpfungserzählung (Gen 1,1-2,4a*)“, in Studien zur Priesterschrift (Tübingen: Mohr, 2008), 94-95, Anm. 12 und Bührer, Am Anfang, 143, Anm. 511; vgl. ebenso Zdravko Stefanovic, „The Great Reversal: Thematic Links between Genesis 2 and 3“, AUSS 32 (1994): 52. Didier Luciani, „Genèse 2,4: théorie documentaire ou analyse narrative?“, NRT 129 (2007): 279-284. Thomas Gudbergesen, „The Unity of Gen 2,4“, SJOT 24 (2010): 235-252.

44 Folgende Argumentation nach Bührer, Am Anfang, 143-144, vgl. ders., „The Relative Dating of the Eden Narrative Gen *2-3“, VT 65 (2015): 375. Witte, Urgeschichte, 54 nennt drei weitere stilistische Unterschiede zwischen Gen 2,4a und 2,4b: 1. Der Wechsel von einer passivischen Konstruktion (,wurden geschaffen“) zu einer aktivischen (,Gott machte"); 2. die unterschiedliche Bildung des Temporalsatzes

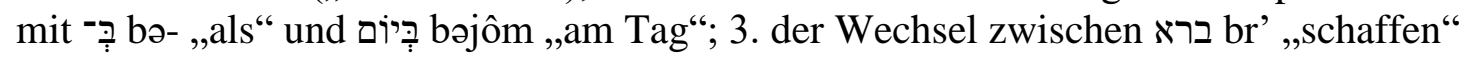
und עשה ,shachen“. Letztere Beobachtung lässt er nicht als Hinweis auf eine literarkritische Schichtung gelten (vgl. Gen 1,1.7; 1,16.21; 1,25.27; 2,3).

45 Vgl. jedoch Otto, „Paradieserzählung“, 187 mit Verweis auf Christoph Dohmen, Schöpfung und Tod: Die Entfaltung theologischer und anthropologischer Konzeptionen in Gen 2/3 (Stuttgart: Katholisches Bibelwerk, 1996), 39-40. 
anderen Teilen der Bibel bezeugt, an den meisten Stellen jedoch determiniert (Ez 8,3; Sach 5,9; 1 Chr 21,16; Jer 10,11). Die Parallelen zu Jesaja 55,9-10 (,Himmel von Erde“ - „,von dem Himmel... die Erde“) und Psalm 148,1.7.13 (,von dem Himmel her... von der Erde her“ - „über Erde und Himmel“, vgl. V. 4.11) überzeugen nicht jeden - dem Psalmisten könnte Gen 2,4 bereits vorgelegen haben ${ }^{46}-$, doch zeigen sie, dass ein einzelner Verfasser durchaus dazu in der Lage ist, Reihenfolge und Determination wiederholter Begriffe zu variieren. Eine weitere Parallele deutet darauf hin, dass ein Namens-Chiasmus im Rahmen einer Toledot/Genealogie kein Zufall ist, wenn nach Abschluss der auf Mose und Aaron hinauslaufenden Genealogie - allerdings ohne ,Toledot“" und ohne Determination - formuliert wird: „Das sind Aaron und Mose... das sind Mose und Aaron“" (Ex 6,26-27). ${ }^{47}$

Walter Bührer, der selbst ,with a probability bordering on certainty ${ }^{648}$ davon ausgeht, dass 2,4 keine literarische Einheit bildet, kommt in seiner forschungsgeschichtlich orientierten Untersuchung dennoch $\mathrm{zu}$ dem Ergebnis: „Die gegen die Einheitlichkeit von Gen 2,4 ausgehend von diesem Vers vorgebrachten Argumente sind für sich genommen $\mathrm{zu}$ wenig stichhaltig“. ${ }^{49}$ Andererseits kann auch die Argumentation für die Einheitlichkeit mit Hilfe von Analogien nicht jeden überzeugen.

Den entscheidenden Ausschlag für eine literarische Einheit könnten von daher die auffälligen Querverbindungen bilden, nicht nur zwischen 2,4a und 1,12,3, sondern eben auch zwischen 2,4b und 1,1-2,3, und entsprechend nicht nur zwischen 2,4b und 2,5-4,26, sondern auch zwischen 2,4a und 2,5-4,26. ${ }^{50}$ Es lässt sich beobachten, dass bis auf ,Toledot" und ,JHWH“ alle Wörter in Gen 2,4 auf den vorausliegenden Bericht zurückgreifen. ${ }^{51}$

\section{Genesis 2,4b im Kontext von Genesis 2,4-3,24}

In Gen 2,4a findet sich die erste Toledot-Formel der Genesis in Verbindung mit Himmel und Erde. Die Toledot-Formel hat in Genesis eine Schaltfunktion, um ein neues Hauptstück einzuleiten: ,,and now I shall tell you in detail what

46 Vgl. ferner Bührer, Am Anfang, 114 skeptisch gegenüber der Parallele Hes 25,12.15 (,machen... Rache“ - „machen... mit der Rache“).

47 Benno Jacob, Das Buch Genesis. Nachdruck der Original-Ausgabe von 1934 (Stuttgart: Calwer, 2000), 76.

48 Bührer, „Relative Dating“, 375.

49 Bührer, Am Anfang, 144, Hervorhebungen im Original.

50 Stordalen, „Genesis 2,4“, 169 redet von „,cross-links“.

51 Fischer, Genesis 1-11, 181. Vgl. Wilfried Warning, „Terminologische Verknüpfungen in der Urgeschichte“, ZAW 114 (2002): 262-269. 
happened.“52 Mit Ausnahme von Gen 2,4 ist „Toledot“ stets mit einem bereits genannten Namen, einer Person, verbunden. ${ }^{53}$

Die Verwendung des Begriffs „Toledot“ gibt Aufschluss darüber, was eine Person hervorgebracht hat, in diesem Fall: Was ist aus (der Schöpfung von) Himmel und Erde hervorgegangen? Hier folgt der Bericht über das Geschehen im Garten Eden. Dieser Paradiesbericht ist im ersten Teil ${ }^{54}$ auch ein Schöpfungsbericht, allerdings in von 1,1-31 abweichender Reihenfolge.

Nach der Toledot-Formel folgt zunächst Genesis 2,4b: Wir werden zurückversetzt zum „Tag“ der Schöpfung von Himmel und Erde. Anschließend folgen andere Schöpfungshandlungen. Wenn Gen 1,1 keine Überschrift oder Zusammenfassung ist, sondern die erste einleitende Schöpfungstat Gottes beschreibt e Schöpfung von Himmel und Erde „in Rohbauform“ -, dann erscheint es ganz natürlich, Gen 2,4b nicht auf das Ganze von 1,1-31 zu beziehen, sondern auf diesen ersten Tag.

Bei diesem Verständnis lässt sich eine auffällige Parallelität zwischen 1,1-2,3 und 2,4-25 erkennen. Zunächst erfolgt die Schöpfungstat $(1,1 ; 2,4)$. Dann folgt als zweiter Schritt eine Beschreibung dessen, was soeben erschaffen wurde. In 1,2 wird die Erde beschrieben: wüst und leer. Alles andere fehlt. Ebenso in 2,5: es gab kein Grün, keinen Regen, keinen Menschen. Schließlich folgt als dritter Schritt der weitere Ausbau der Erde. ${ }^{55}$ Nach der Toledot-Einleitung in 2,4a bildet 2,4b den Anfang der Linie in 2,4-25. Dieser Aufbau spricht dafür, dass sich 2,4b nicht auf das Ganze von 1,1-31 sondern auf den ersten Tag in 1,1-5 bezieht, und zwar auf den ersten Teil dieses Tages: Genesis 1,1.

52 So treffend die Übertragung durch Cassuto, Genesis I, 99. Toledot bedeutet

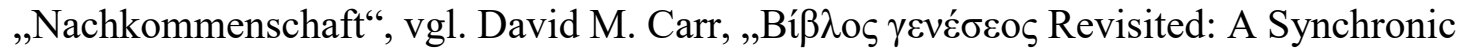
Analysis of Patterns in Genesis as Part of the Torah (Part One)“, ZAW 110 (1998): 159172; Peter Weimar, „Die Toledotformel in der priesterschriftlichen Geschichtsdarstellung“, in Studien zur Priesterschrift (Tübingen: Mohr, 2008), 151184 mit der wichtigsten Literatur, 152, Anm. 6. Sarah Schwartz, „Narrative Toledot Formulae in Genesis: The Case of Heaven and Earth, Noah, and Isaac“, JHS 16 (2016): $1-36$.

53 Nur in Gen 10,1 ist die Formel mit drei Personen verbunden, den drei Söhnen Noahs.

54 Die Paradieserzählung in ihrer Jetztgestalt wird aufgrund der vielen Querbezüge zwischen Genesis 2 und 3 in der Regel als literarische Einheit behandelt, vgl. zur Forschung Arneth, Adams Fall, 97-98; Bührer, Am Anfang, 167-175; Otto, „Paradieserzählung“, 173, Anm. 35. Selbst Christoph Levin, Der Jahwist (Göttingen: Vandenhoeck \& Ruprecht, 1993), 83 isoliert Gen 3 nicht als selbständige Quelle, sondern arbeitet mit einer Ergänzungshypothese. Jüngst betont Fischer, Genesis 1-11, 179-180 wieder den Eigencharakter beider Kapitel.

55 Vgl. die vergleichende Satzanalyse von Bauks, Welt am Anfang, 89, Arneth, Adams Fall, 133 und Weippert, ,Schöpfung am Anfang“, 15-16. 


\section{Genesis 2,4b im Kontext von Genesis 5,1-2 und Numeri 3,1}

Gen 2,4 ist einer von drei Texten, die mit der Toledot-Formel einsetzen und mit dem Ausdruck ,,an dem Tag“ fortfahren (Gen 2,4; Gen 5,1 und Num 3,1). ${ }^{56}$ Nach der Toledot-Formel, die einen neuen Abschnitt einleitet, wird mit dem Ausdruck „an dem Tag“ auf etwas Vergangenes zurückgegriffen, während der Tag zugleich den ersten Teil der daraufhin geschilderten Entwicklung darstellt. In Num 3,1b handelt es sich um den Tag, an welchem JHWH auf dem Berg Sinai die Namen der aaronitische Priester mitteilt. Es ist ein konkreter Tag. Ebenso verweisen Gen 2,4 und 5,1 auf konkrete Tage: In Gen 2,4b handelt es sich um den ersten Schöpfungstag, in Gen 5,1b-2 handelt es sich um den sechsten Schöpfungstag.

In der Form des Rückbezugs, sowie in der Einbettung in den weiteren Verlauf der Erzählung lassen sich enge Parallelen zwischen Gen 2,4 und 5,1-2 erkennen: In Gen 2,4 wendet der Verfasser der Genesis zunächst den Blick zum ersten Tag des Schöpfungsberichtes zurück und zieht dann eine Linie weiter in 2,5-25, wo die Schöpfungstaten JHWHs beschrieben werden, direkt oder indirekt, welche am zweiten, dritten, fünften und sechsten Tag stattfanden ${ }^{57}$ Der

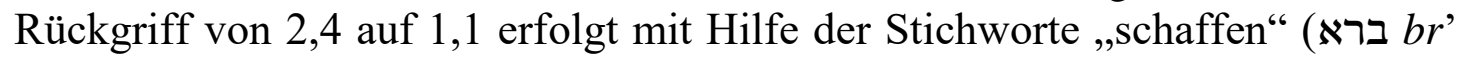

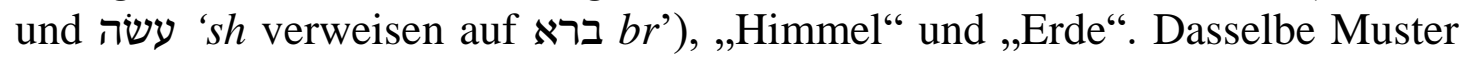
findet sich nun auch im Zusammenhang mit der folgenden Toledot-Formel: In 5,1-2 wendet der Verfasser den Blick zurück auf den sechsten Schöpfungstag und zieht dann die Linie weiter in 5,3-32. Auch hier erfolgt der Rückgriff durch Stichwortverbindungen: ,schaffen“ (ברא br' verweist auf עשה br', Gen 1,26-27), „Adam“ und „Bild“ (5,1), bzw. „Mann und Frau“ (5,2). In Gen 5,1-2 wird zweimal auf den sechsten Tag verwiesen. Dies ist ein konkreter Tag. Diese Parallele stützt die Auslegung von Gen 2,4, welche diesen Vers auf den ersten Tag bezieht. Auch hier handelt es sich um einen konkreten Tag.

\section{BIBLIOGRAPHIE}

Achenbach, Reinhard. Die Vollendung der Tora: Studien zur Rechtsgeschichte des Numeribuches im Kontext von Hexateuch und Pentateuch. BZAR 3. Wiesbaden: Harrassowitz, 2003.

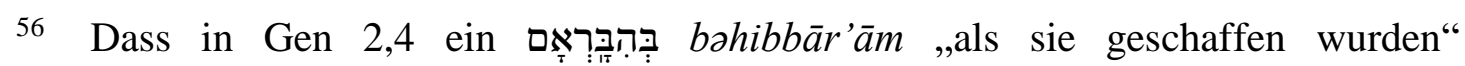
eingeschoben sein könnte, wie Witte, Urgeschichte, 54 und andere nach ihm zu bedenken geben, beeinträchtigt diese Parallelität nur geringfügig und ist für sich genommen kein befriedigender Einwand gegen die Argumentation für die Einheit von 2,4 durch Stordalen, „Genesis 2,4“, 171 aufgrund der deutlichen Parallele zu Gen 5,1 und Num 3,1.

57 Vgl. zur logischen Schwäche einer Argumentation für einen Rückbezug von Gen 2,4-5 auf Tag 3 oder 6, Robert E. Grossmann, „, The Light he Called „Day“““”, MJT 3 (1987): 20. 
Arichea, Daniel C. „Note. The ups and downs of Moses: locating Moses in Exodus 1933.“Bible Translator 40 (1989): 244-246

Arneth, Martin. Durch Adams Fall ist ganz verderbt ...: Studien zur Entstehung der alttestamentlichen Urgeschichte. Göttingen: Vandenhoeck \& Ruprecht, 2007. https://doi.org/10.13109/9783666530807.

Barr, James. Fundamentalismus. München: Chr. Kaiser, 1981. . „Was Everything that God Created really Good? A Question in the First Verse of the Bible“. Seiten 55-65 in God in the Fray: A Tribute to Walter Brueggemann. Herausgegeben von Tod Linafelt und Timothy K. Beal. Minneapolis, MN: Fortress, 1998.

Bauks, Michaela. Die Welt am Anfang: Zum Verhältnis von Vorwelt und Weltentstehung in Gen 1 und in der altorientalischen Literatur. WMANT 74. Neukirchen-Vluyn: Neukirchener, 1997.

Becking, Bob. „Day יום Seiten 221-223 in Dictionary of Deities and Demons in the Bible. Herausgegeben von Karel van der Toorn, u.a. 2. Aufl. Leiden: Brill, 1999. Bergman, Jan, Von Soden, Wolfram und Sæbø, Magne. „„ים yôm“. Seiten 559-586 in Bd. 3 von Theologisches Wörterbuch zum Alten Testament. Herausgegeben von Johannes Botterweck und Helmer Ringgren. Stuttgart: Kohlhammer, 1980.

Blum, Erhard. „Von Gottesunmittelbarkeit zu Gottesähnlichkeit: Überlegungen zur theologischen Anthropologie der Paradieserzählung“. Seiten 1-19 in Textgestalt und Komposition: Exegetische Beiträge zu Tora und Vordere Propheten. Herausgegeben von Wolfgang Oswald. FAT 69. Tübingen: Mohr, 2010. Erstveröffentlichung auf Seiten 9-29 in Gottes Nähe im Alten Testament. Herausgegeben von Eberhardt Gönke und Kathrin Liess. SBS 202, Stuttgart: Katholisches Bibelwerk, 2004.

Booth, Walter M. „Days of Genesis 1: Literal or Nonliteral?“ Journal of the Adventist Theological Society 14 (2003): 101-120.

Brin, Gershon. The Concept of Time in the Bible and the Dead Sea Scrolls. StTDJ 39. Leiden: Brill, 2001. https://doi.org/10.1163/9789047401179 027. . „The Formula X- ימי: Some Characteristics of Historiographical Writing in Israel“". Zeitschrift für die alttestamentliche Wissenschaft 93 (1981): 183-196. https://doi.org/10.1515/zatw.1981.93.2.183.

Budde, Karl. Die Biblische Urgeschichte (Gen. 1-12,5). Gießen: Ricker, 1883. https://doi.org/10.1515/9783111608020.

Bührer, Walter. Am Anfang ...: Untersuchungen zur Textgenese und zur relativchronologischen Einordnung von Gen 1-3. Göttingen: Vandenhoeck \& Ruprecht, 2014. https://doi.org/10.13109/9783666540349. . .,The Relative Dating of the Eden Narrative Gen *2-3“. Vetus Testamentum 65 (2015): 365-376. https://doi.org/10.1163/15685330-12301206.

Cassuto, Umberto. A Commentary on the Book of Genesis. Part I: From Adam to Noah. Genesis I - VI 8. Jerusalem: Magnes, 1998.

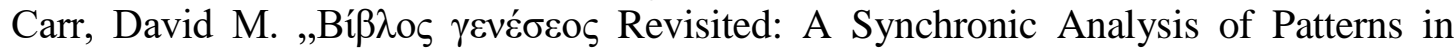
Genesis as Part of the Torah (Part One)“. Zeitschrift für die alttestamentliche Wissenschaft 110 (1998): 159-172. https://doi.org/10.1515/zatw.1998.110.2.159. Reading the Fractures of Genesis: Historic and Literary Approaches. Louisville, KY: Westminster, 1996.

Childs, Brevard S. Myth and Reality in the Old Testament. SBT 27. London: SCM, 1960. 
Chirichigno, Gregory. „The Narrative Structure of Exod 19-24“. Biblica 68 (1987): 457-479.

Collins, Jack. „Discourse Analysis and the Interpretation of Gen 2:4-7“. Westminster Theological Journal 61 (1999): 269-276.

DeVries, Simon John. Yesterday, Today, and Tomorrw: Time and History in the Old Testament. Grand Rapids, MI: Eerdmans, 1975.

Dillmann, August. Die Genesis. KEH. Leipzig: Hirzel, 1892.

Dohmen, Christoph. Schöpfung und Tod: Die Entfaltung theologischer und anthropologischer Konzeptionen in Gen 2/3. SBB 35. Stuttgart: Katholisches Bibelwerk, 1996.

Dozeman, Thomas B. ,Spatial Form in Exod 19:1-8a and in the Larger Sinai Narrative“. Semeia 46 (1989): 87-101.

Fischer, Georg. Genesis 1-11. HThKAT. Freiburg: Herder, 2018.

Fouts, David M. „Selected Lexical and Grammatical Studies in Genesis 1“. Andrews University Seminary Studies 42 (2004): 79-90.

Fretheim, Terence E. „Were the Days of Creation Twenty-Four Hours Long? Yes“. Seiten 12-35 in The Genesis Debate: Persistent Questions About Creation and the Flood. Herausgegeben von Ronald Youngblood. 2. Aufl. Eugene, OR: Wipf \& Stock, 1999.

Gertz, Jan Christian. Das erste Buch Mose. Genesis. Die Urgeschichte: Gen 1-11. ATD. Göttingen: Vandenhoeck \& Ruprecht, 2018. https://doi.org/10.13109/ 9783666570551.

Gesenius, Wilhelm. Handwörterbuch über das Alte Testament, Herausgegeben von Frants Buhl. 17. Aufl. Berlin: Springer, 1962.

Goldingay, John. „KAYYÔM HAZZEH, On This Very Day“; KAYYÔM , On the Very Day'; $K \bar{A} \bar{E} T$,At the Very Time““. Vetus Testamentum 43 (1993): 112-115. https://doi. org/10.1163/156853393X00331.

Grossmann, Robert E. „,The Light he Called „Day“““. Mid-America Journal of Theology 3 (1987): 7-34.

Gudbergsen, Thomas. „The Unity of Gen 2,4“. Scandinavian Journal of the Old Testament 24 (2010): 235-252. https://doi. org/10.1080/09018328.2010.527077.

Gunkel, Hermann. Genesis. HK. Göttingen: Vandenhoeck \& Ruprecht, ${ }^{3} 1922$.

Hilbrands, Walter. „Die Länge der Schöpfungstage: Eine exegetische und rezeptionsgeschichtliche Untersuchung von יוֹם (,Tag“) in Gen 1,1-2,3“. Biblische Notizen. Neue Folge 149 (2011): 3-12.

Hasel, Gerhard F. „The ,Days` of Creation in Genesis 1: Literal ,Days` or Figurative ,Periods/Epochs“ of Time?“. Origins 21 (1994): 5-38.

Hodge, Bryan C. Revisiting the Days of Genesis: A Study of the Use of Time in Genesis 1-11 in Light of Its Ancient Near Eastern and Literary Context. Eugene, OR: Wipf and Stock, 2011.

Houtman, Cornelis. Der Pentateuch: Die Geschichte seiner Erforschung neben einer Auswertung. Kampen: Kok, 1994. . Exodus. Volume 3. Chapters 20 - 40. HCOT. Kampen: Kok, 2000.

Jacob, Benno. Das Buch Genesis. Nachdruck der Original-Ausgabe von 1934. Stuttgart: Calwer, 2000.

Jenni, Ernst. „יוֹ jōm Tag“. Seiten 707-726 in Bd. 1 von Theologisches Handwörterbuch zum Alten Testament Band. Herausgegeben von Ernst Jenni und Claus Westermann. München: Kaiser, ${ }^{5}$ 1994. 707-726. 
104 Riecker and Koorevaar, "Bedeutung," OTE 33/1 (2020): 80-106

Jordan, James B. Creation in Six Days: A Defense of the Traditional Reading of Genesis One. Moscow, ID: Canon, 1999.

Keil, Carl Friedrich. Genesis und Exodus. BC. Leipzig: Dörffling \& Franke, 1878.

Knobel, August. Die Genesis erklärt. KEH. Leipzig: Weidmann, 1852.

Köhler, Ludwig und Baumgartner, Walter. Lexicon in Veteris Testamenti Libros. Leiden: Brill, 1985.

Koorevaar, Hendrik J. „Der Aufbau des Buches Genesis und der literarischtheologische Wert der Entwicklungsformel Toledot“. Seiten 203-217 in Genesis, Schöpfung und Evolution: Beiträge zur Auslegung und Bedeutung des ersten Buches der Bibel, Herausgegeben von Reinhard Junker. Holzgerlingen: SCM Hänssler, 2015.

„The Books of Exodus - Leviticus - Numbers and the Macro-Structural Problem of the Pentateuch“. Seiten 423-453 in The Books of Leviticus and Numbers. Herausgegeben von Thomas Römer. BETL 215. Leuven: Peeters, 2008. . „Steps for Dating the Books of the Pentateuch: a literary and historical canonical approach". Seiten 227-242 in Paradigm Change in Pentateuchal Research. Tagung vom 16.-18. März 2017 an der STH Basel. Herausgegeben von Matthias Armgardt u.a. BZAR 22. Wiesbaden: Harrassowitz, 2019. https://doi.org/10.2307/j.ctvh4zfj2.15.

. „The Torah as One, Three or Five Books: An Introduction to the MacroStructural Problem of the Pentateuch“. Hiphil 3 (2006): 1-19.

. „The Torah Model as the Original Macrostructure of the Hebrew Canon: a Critical Evaluation“. Zeitschrift für die alttestamentliche Wissenschaft 122 (2010): 64-80. https://doi.org/10.1515/zaw.2010.006.

Lanckau, Jörg. „,Tag / Tageszeiten (AT)“, Ohne Seitenangaben in Das

wissenschaftliche Bibellexikon im Internet (WiBiLex). Herausgegeben von

Stefan Alkier u.a. Stuttgart: Deutsche Bibelgesellschaft, 2008. Zitiert am 15.

Mai 2019. Online: http://www.bibelwissenschaft.de/stichwort/32270.

Lavallee, Louis. „Augustine on the Creation Days“. Journal of the Evangelical

Theological Society 32 (1989): 457-464.

Levin, Christoph. Der Jahwist. FRLANT 157. Göttingen: Vandenhoeck \& Ruprecht, 1993. https://doi.org/10.13109/9783666538384.

Lewis, Jack P. „The Days of Creation: An Historical Survey of Interpretation“.

Journal of the Evangelical Theological Society 32 (1989): 433-455.

Luciani, Didier. „Genèse 2,4: théorie documentaire ou analyse narrative?“. La nouvelle revue théologique 129 (2007): 279-284. https://doi.org/10.3917/nrt.292.0279.

Luther, Martin. D. Martin Luthers Werke. Kritische Gesamtausgabe. 42. Band.

Weimar: Böhlaus, 1911.

. Dr. Martin Luthers Auslegung des ersten Buches Mose. Erster Theil. Hg.

Johann Georg Walch, St. Louis, MO: Concordia, 1880.

Marti, Karl. Genesis. KHC. Tübingen: J.C.B. Mohr, 1898.

Marx, Alfred, „La fin du récit sacerdotal de la création: Gn 2,4a ou Gn 2,3?““. Vetus Testamentum 67 (2017) : 581-588. https://doi.org/10.1163/15685330-12341294.

McCabe, Robert V. „A Critique of the Framework Interpretation of the Creation Week". Seiten 211-250 in Coming to Grips with Genesis: Biblical Authority and the Age of the Earth. Herausgegeben von Terry Mortenson und Thane H. Ury. Green Forrest, AR: Master, 2008. 
Nihan, Christophe und Römer, Thomas. „Die Entstehung des Pentateuch: Die aktuelle Debatte“. Seiten 138-164 in Einleitung in das Alte Testament: Die Bücher der Hebräischen Bibel und die alttestamentlichen Schriften der katholischen, protestantischen und orthodoxen Kirche. Herausgegeben von Thomas Römer u.a. Zürich: TVZ, 2013.

Otto, Eckart. Das Deuteronomium im Pentateuch und Hexateuch. Studien zur Literaturgeschichte von Pentateuch und Hexateuch im Lichte des Deuteronomiumrahmens. FAT 30. Tübingen: Mohr Siebeck, 2000.

„Die Paradieserzählung Genesis 2-3: Eine nachpriesterschriftliche Lehrerzählung in ihrem religionshistorischen Kontext“". Seiten 167-192 in ,Jedes Ding hat seine Zeit ... ": Studien zur israelitischen und altorientalischen Weisheit. Diethelm Michel zum 65. Geburtstag. Herausgeben von Anja A. Diesel u.a. BZAW 241. Berlin: De Gruyter, 1996.

Rad, Gerhard von. Das erste Buch Mose. Genesis. ATD. 8. Aufl. Göttingen: Vandenhoeck \& Ruprecht, 1967.

„,Der Tag' im AT“. Seiten 945-949 in Bd. 2 von Theologisches Wörterbuch zum Neuen Testament. Herausgeben von Gerhard Kittel. Stuttgart: Kohlhammer, 1985.

Schüle, Andreas. Die Urgeschichte (Genesis 1-11). ZBK. Zürich: TVZ, 2009. . „Made in the ,Image of God“: The Concepts of Divine Images in Gen 1-3“. Zeitschrift für die alttestamentliche Wissenschaft 117 (2005): 1-20. https://doi.org/10.1515/zatw.2005.117.1.1.

Schwartz, Sarah. „Narrative Toledot Formulae in Genesis: The Case of Heaven and Earth, Noah, and Isaac". Journal of Hebrew Scriptures 16 (2016): 1-36. https://doi.org/10.5508/jhs.2016.v16.a8.

Skinner, John. Genesis. ICC. New York: Scribner, 1910.

Steck, Odil Hannes. Der Schöpfungsbericht der Priesterschrift: Studien zur literarkritischen und überlieferungsgeschichtlichen Problematik von Genesis 1,1-2,4a. FRLANT 115. 2. Aufl. Göttingen: Vandenhoeck \& Ruprecht, 1981.

Stefanovic, Zdravko. „The Great Reversal: Thematic Links Between Genesis 2 and 3“. Andrews University Seminary Studies 32 (1994): 47-56.

Stordalen, Terje, „Genesis 2,4: Restudying a locus classicus“. Zeitschrift für die alttestamentliche Wissenschaft 104 (1992): 163-177. https://doi.org/10.1515 lzatw.1992.104.2.163.

Stroes, H.R. „Does the Day Begin in the Evening or Morning? Some Biblical Observations“. Vetus Testamentum 16 (1966): 460-475. https://doi.org/10.1163/ $156853366 \times 00287$.

Verhoef, Pieter A. „יi" “. Seiten 419-424 in Bd. 2 von New International Dictionary of Old Testament Theology and Exegesis. Herausgegeben von Willem A. VanGemeren. Carlisle: Paternoster, 1997.

Vervenne, Marc. „Genesis 1,1-2,4. The Compositional Texture of the Priestly Overture to the Pentateuch", Seiten 35-79 in Studies in the Book of Genesis: Literature, Redaction and History. Herausgegben von André Wénin. BeThL 155, Leuven: Peeters, 2001.

Wadholm, Rick. „What Is In A Day?: The Theological Significance of Yôm in Genesis 1“. Didaskalia 24 (2015): 75-98.

Waltke, Bruce K. und O'Conner, Michael P. An Introduction to Biblical Hebrew Syntax. Winona Lake, IN: Eisenbrauns, 1990. 
106 Riecker and Koorevaar, "Bedeutung," OTE 33/1 (2020): 80-106

Warning, Wilfried. „Terminologische Verknüpfungen in der Urgeschichte“, Zeitschrift für die alttestamentliche Wissenschaft 114 (2002): 262-269. https://doi.org/ 10.1515/zatw.2002.015.

Weimar, Peter. „Die Toledotformel in der priesterschriftlichen Geschichtsdarstellung“, Seiten 151-184 in Studien zur Priesterschrift. FAT 56. Tübingen: Mohr Siebeck, 2008. Erstveröffentlichung in Biblische Zeitschrift 18 (1974): 65-93.

. „Struktur und Gestalt der priesterschriftlichen Schöpfungserzählung (Gen 1,1-2,4a*)“, Seiten 91-134 in Studien zur Priesterschrift. FAT 56. Tübingen: Mohr Siebeck, 2008. Erstveröffentlichung auf Seiten 803-843 in Ex Mesopotamia et Syria Lux. Festschrift für Manfried Dietrich zu seinem 65. Geburtstag. Herausgegeben von Oswald Loretz u.a. AOAT 281. Münster: Ugarit, 2002.

Weippert, Manfred. „Schöpfung am Anfang oder Anfang der Schöpfung? Noch einmal zu Syntax und Semantik von Gen 1,1-3“. Theologische Zeitschrift 60 (2004): 522.

Wellhausen, Julius. Prolegomena zur Geschichte Israels. 6. Aufl. Berlin: De Gruyter, 1927.

Wenham, Gordon J. Genesis 1-15. WBC. Waco, TX: Word, 1987.

. „The Priority of P“. Vetus Testamentum 49 (1999): 240-258. https://doi.org/ $10.1163 / 156853399774229000$.

Westermann, Claus. Genesis. Bd. 1: Genesis 1-11. BKAT. 2. Aufl. Neukirchen-Vluyn: Neukirchener, 1976.

Wiles, Maurice F. God's Action in the World. The Bampton Lectures for 1986. London: SCM, 1986.

Wilch, John R. Time and Event: An Exegetical Study of the use of 'êth in the Old Testament in Comparison to other Temporal Expressions in Clarification of the Concept of Time. Leiden: Brill, 1969.

Witte, Markus. Die biblische Urgeschichte: Redaktions- und theologiegeschichtliche Beobachtungen zu Genesis 1,1 - 11,26. Berlin: De Gruyter, 1998. https://doi.org/10.1515/9783110804614.

Young, Edward J. „The Days of Genesis. First Article“, Westminster Theological Journal 25 (1963): 1-34.

„The Days of Genesis. Second Article“. Westminster Theological Journal 25 (1963): 143-171.

Siegbert Riecker is External Instructor in Old Testament at the Evangelische Theologische Faculteit in Leuven, Belgium. Email: siegbert.riecker@etf.edu. ORCID: https://orcid.org/0000-0003-3068-475X.

Hendrik Koorevaar is Professor Emeritus for the Old Testament at the Evangelische Theologische Faculteit in Leuven, Belgium. Email: hendrik.koorevaar@gmail.com. Website: https://etfleuven.academia.edu/Hendri kKoorevaar and https://nl.wikipedia.org/wiki/Hendrik_Koorevaar. ORCID: https://orcid.org/0000-0002-1962-8557. 